\title{
Generalized Hamming weights of $q$-ary Reed-Muller codes
}

\author{
Petra Heijnen and Ruud Pellikaan * \\ Appeared in IEEE Trans. Inform. Theory, \\ vol. 44, pp. 181-196, Jan. 1998.
}

\begin{abstract}
The order bound on generalized Hamming weights is introduced in a general setting of codes on varieties which comprises both the one point geometric Goppa codes as the $q$-ary Reed-Muller codes. For the latter codes it is shown that this bound is sharp and that they satisfy the double chain condition.
\end{abstract}

\section{Introduction}

In terms of polynomials the problem of finding the generalized Hamming weights of $q$-ary Reed-Muller codes is equivalent to Problem 7.1 of [39]:

Let $f_{1}, \ldots, f_{r}$ be linearly independent polynomials in $m$ variables of degree $u$ or less with coefficients in $\mathbb{F}_{q}$, the finite field of $q$ elements.

What is the maximum possible number of solutions in $\mathbb{F}_{q}^{m}$ of the system $f_{1}=\cdots=f_{r}=0$ ?

Let $n=q^{m}$. Let $P_{1}, \ldots, P_{n}$ be an enumeration of the points of $\mathbb{F}_{q}^{m}$. The ring of polynomials in $m$ variables with coefficients in $\mathbb{F}_{q}$ is denoted by $\mathbb{F}_{q}\left[X_{1}, \ldots, X_{m}\right]$. Consider the evaluation map

$$
\text { ev }: \mathbb{F}_{q}\left[X_{1}, \ldots, X_{m}\right] \longrightarrow \mathbb{F}_{q}^{n}
$$

\footnotetext{
*Both authors are from the Department of Mathematics and Computing Science, Eindhoven University of Technology, P.O. Box 513, 5600 MB Eindhoven, The Netherlands.
} 
defined by $e v(f)=\left(f\left(P_{1}\right), \ldots, f\left(P_{n}\right)\right)$. The $q$-ary Reed-Muller $(\mathrm{RM})$ code $\mathcal{R M}_{q}(u, m)$ of order $u$ in $m$ variables is the image of this evaluation map of all polynomials of degree $u$ or less. The polynomials which evaluate to the zero word form the ideal generated by $X_{i}^{q}-X_{i}$ for $i=1, \ldots, m$. If the polynomial $f$ is evaluated to the word $\mathbf{c}$, then the number of zeros of $f$ in $\mathbb{F}_{q}^{m}$ is equal to $n-w t(\mathbf{c})$. If the polynomials $f_{1}, \ldots, f_{r}$ are evaluated to the words $\mathbf{c}_{1}, \ldots, \mathbf{c}_{r}$, then the number of common zeros of these polynomials is equal to $n$ minus the size of the support of the linear code generated by $\mathbf{c}_{1}, \ldots, \mathbf{c}_{r}$. The support of a code $C$ is defined by

$$
\operatorname{supp}(C)=\left\{i: c_{i} \neq 0 \text { for some } \mathbf{c} \in C\right\}
$$

The support weight or effective length of $C$ is the number of elements of its support.

The $r$ th generalized Hamming weight (GHW) or $r$ th minimum support weight of a linear code $C$ is defined by

$$
d_{r}(C)=\min \{|\operatorname{supp}(D)|: D \text { is a linear subcode of } C \text { and } \operatorname{dim}(D)=r\} .
$$

The RM codes were defined in $[32,28]$ for the binary case and this was generalized to arbitrary $q$ in $[7,18,43]$.

Generalized Hamming weights were introduced in the study [15, 24] on the weight distribution of codes over extensions of $\mathbb{F}_{q}$. The concept was rediscovered in [41] where the problem of the GHW's of the binary RM codes was solved. In [1] the GHW's of the ternary RM codes was computed. An equivalent definition was found for projective systems in projective space in $[38,39]$. The generalized weight enumerator was defined in [25] and a Macwilliams identity was proved in [34].

The connection between the trellis or state complexity of a code and its GHW's was made in $[20,21]$. The sequence of GHW's is called the length/dimension profile (LDP) in [13]. The inverse function is called the dimension/length profile (DLP). The DLP bound on the state complexity becomes an equality if the code satisfies the double chain condition [8, 14, 20, 42].

A code $C$ satisfies the chain condition if there is an increasing sequence $\left(D_{r}\right)$ of subcodes of $C$ such that $D_{r} \subset D_{r+1}, \operatorname{dim}\left(D_{r}\right)=r$ and the effective length of $D_{r}$ is $d_{r}(C)$ for all $r$. Or equivalently, there exists a permutation of the coordinates and there is an increasing sequence $\left(D_{r}\right)$ of subcodes of $C$ such that $D_{r} \subset D_{r+1}, \operatorname{dim}\left(D_{r}\right)=r$ and $\operatorname{supp}\left(D_{r}\right)=\left\{1,2, \ldots, d_{r}\right\}$ for all $r$. 
A code $C$ satisfies the two way or double chain condition if there exists a permutation of the coordinates and there are increasing sequences $\left(D_{r}^{L}\right)$ and $\left(D_{r}^{R}\right)$ of subcodes of $C$ such that $D_{r}^{L} \subset D_{r+1}^{L}, \operatorname{dim}\left(D_{r}^{L}\right)=r$ and such that $D_{r}^{R} \subset D_{r+1}^{R}, \operatorname{dim}\left(D_{r}^{R}\right)=r$ and $\operatorname{supp}\left(D_{r}^{L}\right)=\left\{1,2, \ldots, d_{r}\right\}$ and $\operatorname{supp}\left(D_{r}^{R}\right)=\left\{n+1-d_{r}, n+2-d_{r}, \ldots, n\right\}$ for all $r$.

A general setting will be given where our method applies. This is taken from [16] and originates from $[11,12]$. Consider a geometric object $\mathcal{X}$ with a subset $\mathcal{P}$ consisting of $n$ points which are enumerated by $P_{1}, \ldots, P_{n}$. Suppose that we have a vector space $L$ over $\mathbb{F}_{q}$ of functions on $\mathcal{X}$ such that $f\left(P_{i}\right) \in \mathbb{F}_{q}$ for all $i$ and $f \in L$. In this way one has an evaluation map

$$
e v_{\mathcal{P}}: L \longrightarrow \mathbb{F}_{q}^{n}
$$

which is defined by $e v_{\mathcal{P}}(f)=\left(f\left(P_{1}\right), \ldots, f\left(P_{n}\right)\right)$. If this evaluation map is linear, then its image $E$ and its dual $C$ are linear codes. The notion of an order function on the space of functions on $\mathcal{X}$ gives sequences of vector spaces $L(l)$, evaluation codes $E(l)$ and their duals $C(l)$, where $l$ is a parameter denoting the dimension of $L(l)$. These codes comprise the one point geometric Goppa codes and the RM codes. In the first case $\mathcal{X}$ is an algebraic curve defined over $\mathbb{F}_{q}, \mathcal{P}$ is a set of rational points on $\mathcal{X}$ and $L$ is a vector space of rational functions which have only poles at a fixed point $P$ with an upper bound on the pole order at $P$. In the second case $\mathcal{X}$ is the affine space of dimension $m, \mathcal{P}$ is the set of all $q^{m}$ rational points of $\mathcal{X}$ and $L$ is the vector space of all polynomials in $m$ variables with coefficients in $\mathbb{F}_{q}$ of degree at most $u$.

The decoding of these codes can be done by majority voting of unknown syndromes $[10,12,16,17,30]$.

In this paper the order bound on the generalized Hamming weights is defined and in detail studied for the RM codes over all finite fields. It turns out that the order bound is sharp in this case. The shift bound as defined in [30] is tight for the minimum distance of RM codes but does not give the right answer for the higher Hamming weights.

A direct consequence of our methods is that the RM codes satisfy the double chain condition. This gives the state complexity of these codes as done in the binary case by $[20,21]$. 
In Section 2 the definition of an order function is given. The order bound on the generalized Hamming weights is given in Section 3. The theory is applied to Reed-Muller codes in Section 4. Some combinatorics of extremal poset theory is applied to get a formula for the higher weights of RM codes in Section 5. Another formula is derived in Section 6. We conclude with some remarks and open problems.

\section{Order functions and higher weights}

In this section the notion of an order function will be defined. It has its origin in valuation theory [9] and the theory of Gröbner bases [4, 6].

In this paper $\mathbb{F}$ denotes a field, $\mathbb{F}_{q}$ the finite field with $q$ elements. An $\mathbb{F}$ algebra will be a commutative ring with a unit that contains $\mathbb{F}$ as a unitary subring. $\mathbb{N}$ denotes the positive integers and $\mathbb{N}_{0}$ the nonnegative integers.

The dimension of polynomials in one variable of degree at most $d$ is equal to $d+1$. This is no longer the case for polynomials in several variables. Decreasing sequences of codes $(C(l) \mid l \in \mathbb{N})$ will be introduced. The code $C(l)$ is defined by parity checks with words that are evaluations of functions in a certain $\mathbb{F}_{q}$-algebra of a given order. For inductive arguments for bounds on the generalized Hamming weights it is necessary that the dimension of $C(l)$ is at most one more than the dimension of $C(l+1)$.

A way to obtain this objective for the ring of polynomials in two variables is to define the order by looking at the degree firstly and the lexicographic order on the exponents $(i, j)$ of the monomial $X^{i} Y^{j}$ secondly. This order $\prec$ looks like

$$
1 \prec Y \prec X \prec Y^{2} \prec X Y \prec X^{2} \prec Y^{3} \prec X Y^{2} \ldots
$$

Notice that this order respects multiplication of monomials. This is formalized as follows.

Definition 2.1 Let $R=\mathbb{F}\left[X_{1}, \ldots, X_{m}\right]$. Suppose that $\prec$ is a total order on the set of monomials in the variables $X_{1}, \ldots, X_{m}$ such that for all monomials $M_{1}, M_{2}$, and $M$ the following holds

$(R .1) \quad$ If $M \neq 1$, then $1 \prec M$,

$(R .2) \quad$ If $M_{1} \prec M_{2}$, then $M M_{1} \prec M M_{2}$. 
Then $\prec$ is called a reduction, term or admissible order on the monomials [4, 6].

The multi-index notation is used for monomials. That means $X^{\alpha}=\prod_{s=1}^{m} X_{s}^{\alpha_{s}}$ if $\alpha=\left(\alpha_{1}, \ldots, \alpha_{m}\right)$. The degree of $X^{\alpha}$ and $\alpha$ is defined by

$$
\operatorname{deg}\left(X^{\alpha}\right)=\operatorname{deg}(\alpha)=\sum_{s=1}^{m} \alpha_{s} .
$$

Giving a reduction order on monomials in $m$ variables is the same as giving a total order on $\mathbb{N}_{0}^{m}$ such that, for all $\alpha_{1}, \alpha_{2}$, and $\alpha$ in $\mathbb{N}_{0}^{m}$, the following holds

(E.1) If $\alpha \neq 0$, then $0 \prec \alpha$,

(E.2) If $\alpha_{1} \prec \alpha_{2}$, then $\alpha+\alpha_{1} \prec \alpha+\alpha_{2}$.

We use $\prec$ both for monomials and exponents.

Example 2.2 The lexicographic order $\prec_{L}$ is defined by

$$
X^{\alpha} \prec_{L} X^{\beta} \Leftrightarrow \alpha_{1}=\beta_{1}, \ldots, \alpha_{l-1}=\beta_{l-1} \text { and } \alpha_{l}<\beta_{l} \text { for some } l \text {. }
$$

The lexicographic order is a reduction order. See [6]. For $m=2$ with $X=X_{1}$ and $Y=X_{2}$ the start of the lexicographic order looks like

$$
\begin{array}{ccrrrrrrrrrrrr}
1 & \prec & Y & \prec & Y^{2} & \prec & \cdots & \prec & Y^{j} & \prec & Y^{j+1} & \prec & \cdots \\
X & \prec & X Y & \prec & X Y^{2} & \prec & \cdots & \prec & X Y^{j} & \prec & X Y^{j+1} & \prec & \cdots \\
X^{2} & \prec & \cdots & & & & & & & & & &
\end{array}
$$

Hence $X^{i+1}$ is the supremum of the set $\left\{X^{i} Y^{j} \mid j \in \mathbb{N}_{0}\right\}$. If $m \geq 2$, then the lexicographic order is not isomorphic with the positive integers.

Example 2.3 The graded lexicographic order $\prec_{D}$ is defined by

$$
X^{\alpha} \prec_{D} X^{\beta} \Leftrightarrow \operatorname{deg}\left(X^{\alpha}\right)<\operatorname{deg}\left(X^{\beta}\right) \text { or } \operatorname{deg}\left(X^{\alpha}\right)=\operatorname{deg}\left(X^{\beta}\right), X^{\alpha} \prec_{L} X^{\beta} \text {. }
$$

The graded lexicographic order is a reduction order [6] which is isomorphic with the positive integers. This section started with the two variable case as an example.

The order is extended to a function on all polynomials in the following way. Let $\prec$ be a reduction order which is isomorphic with the positive integers. 
Let $f_{1}, f_{2}, \ldots$ be an enumeration of the set of monomials such that $f_{i} \prec f_{i+1}$ for all $i$. The monomials are a basis of $\mathbb{F}\left[X_{1}, \ldots, X_{m}\right]$ over $\mathbb{F}$. Hence every nonzero polynomial $f$ can be written in a unique way as

$$
f=\sum_{i=1}^{j} \lambda_{i} f_{i},
$$

where $\lambda_{i} \in \mathbb{F}$ for all $i$, and $\lambda_{j} \neq 0$. Then $f_{j}$ is called the leading monomial of $f$. Define a function

$$
\rho: \mathbb{F}\left[X_{1}, \ldots, X_{m}\right] \longrightarrow \mathbb{N}_{0} \cup\{-\infty\},
$$

by $\rho(0)=-\infty$ and $\rho(f)=j-1$ where $j$ is the smallest positive integer such that $f$ can be written as a linear combination of the first $j$ monomials. It is not difficult to show that $\rho$ satisfies the following conditions

(O.0) $\rho(f)=-\infty$ if and only if $f=0$

(O.1) $\quad \rho(\lambda f)=\rho(f)$ for all nonzero $\lambda \in \mathbb{F}$

$(O .2) \quad \rho(f+g) \leq \max \{\rho(f), \rho(g)\}$ and equality holds when $\rho(f)<\rho(g)$.

$(O .3)$ If $\rho(f)<\rho(g)$ and $h \neq 0$, then $\rho(f h)<\rho(g h)$

(O.4) If $\rho(f)=\rho(g)$, then there exists a nonzero $\lambda \in \mathbb{F}$ such that $\rho(f-\lambda g)<\rho(g)$.

for all $f, g, h \in R$. Here $-\infty<n$ for all $n \in \mathbb{N}_{0}$. The properties of the function $\rho$ are captured in the following definition.

Definition 2.4 Let $R$ be an $\mathbb{F}$-algebra. An order function on $R$ is a map

$$
\rho: R \longrightarrow \mathbb{N}_{0} \cup\{-\infty\}
$$

that satisfies conditions $(O .0), \ldots,(O .4)$.

\section{The order bound on the generalized weights}

In this section a sequence $(C(l): l \in \mathbb{N})$ of codes is defined by means of an $\mathbb{F}_{q}$-algebra $R$ together with an order function $\rho$ on $R$ and a morphism of $\mathbb{F}_{q}$-algebras $\varphi: R \rightarrow \mathbb{F}_{q}^{n}$. The order bound $d_{r}(l)$ is defined and it is shown 
that it is a lower bound on the $r$ th generalized Hamming weight of $C(l)$.

Let $R$ be an $\mathbb{F}_{q}$-algebra with an order function $\rho$. So let $\left\{f_{i}: i \in \mathbb{N}\right\}$ be a basis of $R$ over $\mathbb{F}_{q}$ such that $\rho\left(f_{i}\right)<\rho\left(f_{i+1}\right)$ for all $i \in \mathbb{N}$, and for all nonzero $f \in R$ there exists a $j$ with $\rho(f)=\rho\left(f_{j}\right)$. The existence of such a basis can be shown in general $[16,30]$, but this fact is not needed in this paper since we specialize to the ring $R=\mathbb{F}_{q}\left[X_{1}, \ldots, X_{m}\right]$ which has a basis of monomials with the above mentioned property. Let $L(l)$ be the vector space generated by $f_{1}, \ldots, f_{l}$. Hence for all nonzero $f \in R$ we have that $\rho(f)=\rho\left(f_{l}\right)$ if and only if $l$ is the smallest integer such that $f \in L(l)$. Let $l(i, j)$ be the smallest positive integer $l$ such that $f_{i} f_{j} \in L(l)$. So $l(i, j)<l(i+1, j)$ for all $i, j \in \mathbb{N}$.

The standard innerproduct is defined by $\mathbf{a} \cdot \mathbf{b}=\sum a_{i} b_{i}$ for $\mathbf{a}=\left(a_{1}, \ldots, a_{n}\right)$ and $\mathbf{b}=\left(b_{1}, \ldots, b_{n}\right)$. The coordinatewise multiplication $*$ on $\mathbb{F}_{q}^{n}$ is defined by $\mathbf{a} * \mathbf{b}=\left(a_{1} b_{1}, \ldots, a_{n} b_{n}\right)$. The vector space $\mathbb{F}_{q}^{n}$ becomes an $\mathbb{F}_{q}$-algebra with this multiplication.

Let

$$
\varphi: R \longrightarrow \mathbb{F}_{q}^{n},
$$

be a morphism of $\mathbb{F}_{q}$-algebras, that means $\varphi$ is $\mathbb{F}$-linear and

$$
\varphi(f g)=\varphi(f) * \varphi(g)
$$

Let $\mathbf{h}_{i}=\varphi\left(f_{i}\right)$. Define

$$
C(l)=\left\{\mathbf{c} \in \mathbb{F}_{q}^{n}: \mathbf{c} \cdot \mathbf{h}_{i}=0 \text { for all } i \leq l\right\} .
$$

In this paper only those algebra morphisms $\varphi$ will be considered that are surjective. Then there exists an $N$ such that $\mathbf{h}_{1}, \ldots, \mathbf{h}_{N}$ generate $\mathbb{F}_{q}^{n}$. Hence $C(l)=0$ for all $l \geq N$.

Definition 3.1 Let $\mathbf{y} \in \mathbb{F}_{q}^{n}$. Consider the syndromes

$$
s_{i}(\mathbf{y})=\mathbf{y} \cdot \mathbf{h}_{i} \quad \text { and } \quad s_{i j}(\mathbf{y})=\mathbf{y} \cdot\left(\mathbf{h}_{i} * \mathbf{h}_{j}\right) .
$$

Then $S(\mathbf{y})=\left(s_{i j}(\mathbf{y}): 1 \leq i, j \leq N\right)$ is the matrix of syndromes of $\mathbf{y}$.

Lemma 3.2 Let $\mathbf{y} \in \mathbb{F}_{q}^{n}$. Let $D(\mathbf{y})$ be the $n \times n$ diagonal matrix with $\mathbf{y}$ on the diagonal. Let $H$ be the $N \times n$ matrix with $\mathbf{h}_{i}$ on the ith row. Then

$$
S(\mathbf{y})=H D(\mathbf{y}) H^{T},
$$


Proof. The matrix of syndromes $S(\mathbf{y})$ is equal to $H D(\mathbf{y}) H^{T}$, since

$$
s_{i j}(\mathbf{y})=\mathbf{y} \cdot\left(\mathbf{h}_{i} * \mathbf{h}_{j}\right)=\sum_{l=1}^{n} y_{l} h_{i l} h_{j l},
$$

where $h_{i l}$ is the $l$ th entry of $\mathbf{h}_{i}$.

Definition 3.3 We define the image of $S$ with respect to $U$ as $\operatorname{Im}(S, U)=\left\{\mathbf{y} \in \mathbb{F}_{q}^{N}:\right.$ there exist $\mathbf{u} \in U$ and $\mathbf{x} \in \mathbb{F}_{q}^{N}$ such that $\left.\mathbf{x} S(\mathbf{u})=\mathbf{y}\right\}$ and its linear span is denoted by $\operatorname{Im}^{*}(S, U)$. The image of $D$ with respect to $U$ is defined by

$\operatorname{Im}(D, U)=\left\{\mathbf{w} \in \mathbb{F}_{q}^{n}:\right.$ there exist $\mathbf{u} \in U$ and $\mathbf{v} \in \mathbb{F}_{q}^{n}$ such that $\left.\mathbf{v} D(\mathbf{u})=\mathbf{w}\right\}$ and its linear span is denoted by $\operatorname{Im}^{*}(D, U)$.

Remark 3.4 The images $\operatorname{Im}(S, U)$ and $\operatorname{Im}(D, U)$ are in general not linear spaces. Take for instance $q=2$ and $U=\langle(1,1,0),(0,1,1)\rangle$. Then $\operatorname{Im}(D, U)=\mathbb{F}_{2}^{3} \backslash\{(1,1,1)\}$.

\section{Lemma 3.5}

1) $\operatorname{Im}^{*}(S, U) \cong \operatorname{Im}^{*}(D, U)$,

2) $\operatorname{Im}^{*}(D, U)=\left\langle\mathbf{e}_{j}: j \in \operatorname{supp}(U)\right\rangle$,

3) $\operatorname{dim}\left(\operatorname{Im}^{*}(S, U)\right)=|\operatorname{supp}(U)|$.

\section{Proof.}

1) Consider the linear map $\eta: \mathbb{F}_{q}^{n} \rightarrow \mathbb{F}_{q}^{N}$ with matrix $H^{T}$, so $\eta(\mathbf{w})=\mathbf{w} H^{T}$. We claim that $\eta(\operatorname{Im}(D, U))=\operatorname{Im}(S, U)$.

Let $\mathbf{w} \in \operatorname{Im}(D, U)$, then there exists a $\mathbf{v} \in \mathbb{F}_{q}^{n}$ such that $\mathbf{w}=\mathbf{v} D(\mathbf{u})$. The rows of $H$ generate $\mathbb{F}_{q}^{n}$, since $\varphi$ is surjective. So $\mathbf{v}=\mathbf{x} H$ for some $\mathbf{x} \in \mathbb{F}_{q}^{N}$. Hence $\eta(\mathbf{w})=\mathbf{w} H^{T}=\mathbf{v} D(\mathbf{u}) H^{T}=\mathbf{x} H D(\mathbf{u}) H^{T}=\mathbf{x} S(\mathbf{u}) \in \operatorname{Im}(S, U)$, where the last equality is stated in Lemma 3.2.

Suppose $\mathbf{y} \in \operatorname{Im}(S, U)$. Then there exists an $\mathbf{x} \in \mathbb{F}_{q}^{N}$ and a $\mathbf{u} \in U$ such that $\mathbf{y}=\mathbf{x} S(\mathbf{u})$. We can write $S(\mathbf{u})=H D(\mathbf{u}) H^{T}$, so $\mathbf{y}=\mathbf{x} H D(\mathbf{u}) H^{T}$. Let $\mathbf{v}=\mathbf{x} H$, then $\mathbf{v} \in \mathbb{F}_{q}^{n}$. Let $\mathbf{w}=\mathbf{v} D(\mathbf{u})$, then $\mathbf{w} \in \operatorname{Im}(D, U)$ and $\eta(\mathbf{w})=\mathbf{w} H^{T}=\mathbf{y}$. This proves the claim.

The map $\eta$ is injective, since $H$ has rank $n$. Therefore $\operatorname{Im}^{*}(S, U) \cong \operatorname{Im}^{*}(D, U)$ under $\eta$. 
2) Let $\mathbf{y} \in \operatorname{Im}(D, U)$. Then there is a $\mathbf{u} \in U$ and an $\mathbf{x} \in \mathbb{F}_{q}^{n}$, such that $\mathbf{y}=D(\mathbf{u}) \mathbf{x}=\mathbf{u} * \mathbf{x}$. So $y_{j}=u_{j} x_{j}$, and $y_{j}=0$ if $j \notin \operatorname{supp}(U)$. Hence $\left\langle\mathbf{e}_{j}: j \in \operatorname{supp}(U)\right\rangle$ is a basis of $\operatorname{Im}^{*}(D, U)$, where $\mathbf{e}_{j}$ is the $j$ th standard basis vector of length $n$, that is to say the $i$ th coordinate of $\mathbf{e}_{j}$ is one if $i=j$ and zero otherwise.

3) This follows directly from 1) and 2).

Remark 3.6 One can show that $\operatorname{Im}(D, U)=\operatorname{Im}^{*}(D, U)$ and $\operatorname{Im}(S, U)=$ $\operatorname{Im}^{*}(S, U)$ if $q>|\operatorname{supp}(U)|$.

Lemma 3.7 Let $U$ be a linear subspace of $C(l)$ of dimension $r$ with $r>0$. Then there exists a unique $r$-tuple $\left(l_{1}, \ldots, l_{r}\right)$ such that $l \leq l_{1}<\cdots<l_{r}<N$ and $\operatorname{dim}\left(U \cap C\left(l_{i}+1\right)\right)=\operatorname{dim}\left(U \cap C\left(l_{i}\right)\right)-1$ for $i=1, \ldots, r$.

Proof. Let $U(m)=U \cap C(m)$. Then $(U(m): m \in \mathbb{N})$ is a decreasing chain of subspaces of $U$. Furthermore $U(l)=U$ has dimension $r>0, U(m)=0$ for $m \geq N$ and

$$
\operatorname{dim} U(m)-1 \leq \operatorname{dim} U(m+1) \leq \operatorname{dim} U(m) .
$$

So there are exactly $r$ values for $m$ such that $l \leq m<N$ and $\operatorname{dim} U(m+1)=$ $\operatorname{dim} U(m)-1$, which are denoted by $l_{1}<\cdots<l_{r}$.

Definition 3.8 The $\left(l_{1}, \ldots, l_{r}\right)$ is called the associated $r$-tuple of the subspace $U$ of $C(l)$ of dimension $r$.

Definition 3.9 Define the sets $N(l)$ and $A(l)$ by

$$
\begin{aligned}
& N(l)=\left\{(i, j) \in \mathbb{N}^{2}: l(i, j)=l+1\right\} \\
& A(l)=\{i \in \mathbb{N}: l(i, j)=l+1 \text { for some } j\} .
\end{aligned}
$$

Define for $l_{1}<\cdots<l_{r}$ the set $A\left(l_{1}, \ldots, l_{r}\right)$ by

$$
A\left(l_{1}, \ldots, l_{r}\right)=\cup_{t=1}^{r} A\left(l_{t}\right) .
$$

Let $a\left(l_{1}, \ldots, l_{r}\right)$ denote the number of elements of $A\left(l_{1}, \ldots, l_{r}\right)$.

\section{Lemma 3.10}

1) If $\mathbf{y} \in C(l)$ and $l(i, j) \leq l$, then $s_{i j}(\mathbf{y})=0$.

2) If $\mathbf{y} \in C(l) \backslash C(l+1)$ and $l(i, j)=l+1$, then $s_{i j}(\mathbf{y}) \neq 0$. 


\section{Proof.}

1) Let $\mathbf{y} \in C(l)$. If $l(i, j) \leq l$, then $f_{i} f_{j} \in L(l)$. So $\mathbf{h}_{i} * \mathbf{h}_{j}=\varphi\left(f_{i} f_{j}\right)$ is an element of $\varphi(L(l))$, which is the dual of $C(l)$. Hence $s_{i j}(\mathbf{y})=\mathbf{y} \cdot\left(\mathbf{h}_{i} * \mathbf{h}_{j}\right)=0$. 2) Let $\mathbf{y} \in C(l) \backslash C(l+1)$. If $l(i, j)=l+1$, then $f_{i} f_{j} \in L(l+1) \backslash L(l)$. So $f_{i} f_{j} \equiv \mu f_{l+1}$ modulo $L(l)$ for some nonzero $\mu \in \mathbb{F}_{q}$. Hence $\mathbf{h}_{i} * \mathbf{h}_{j} \equiv \mu \mathbf{h}_{l+1}$ modulo $\varphi(L(l))$. Now $\mathbf{y} \notin C(l+1)$, so $s_{l+1}(\mathbf{y}) \neq 0$. Therefore $s_{i j}(\mathbf{y}) \neq 0$

Lemma 3.11 If $t=|N(l)|$ and $\left(i_{1}, j_{1}\right), \ldots,\left(i_{t}, j_{t}\right)$ is an enumeration of the elements of $N(l)$ in increasing order with respect to the lexicographic order on $\mathbb{N}^{2}$, then $i_{1}<\cdots<i_{t}$ and $j_{t}<\cdots<j_{1}$. In particular $N(l)$ and $A(l)$ have the same number of elements. If moreover $\mathbf{y} \in C(l) \backslash C(l+1)$, then

$$
s_{i_{r} j_{s}}(\mathbf{y})= \begin{cases}0 & \text { if } \quad r<s \\ \text { not zero } & \text { if } \quad r=s .\end{cases}
$$

Proof. The sequence $\left(i_{1}, j_{1}\right), \ldots,\left(i_{t}, j_{t}\right)$ is ordered in such a way that $i_{1} \leq$ $\ldots \leq i_{t}$ and $j_{r}<j_{r+1}$ if $i_{r}=i_{r+1}$. If $i_{r}=i_{r+1}$, then $j_{r}<j_{r+1}$. So

$$
l+1=l\left(i_{r}, j_{r}\right)<l\left(i_{r}, j_{r+1}\right)=l\left(i_{r+1}, j_{r+1}\right)=l+1,
$$

which is a contradiction. Hence the sequence $i_{1}, \ldots, i_{t}$ is strictly increasing. A similar argument shows that $j_{s+1}<j_{s}$ for all $s<t$.

Let $\mathbf{y} \in C(l)$. If $r<s$, then $l\left(i_{r}, j_{s}\right)<l\left(i_{s}, j_{s}\right)=l+1$. Lemma 3.10 implies that $s_{i_{r} j_{s}}(\mathbf{y})=0$.

Moreover, let $\mathbf{y} \notin C(l+1)$. If $r=s$, then $l\left(i_{r}, j_{s}\right)=l+1$. Lemma 3.10 implies that $s_{i_{r} j_{s}}(\mathbf{y}) \neq 0$.

Proposition 3.12 Let $U$ be a linear subspace of $C(l)$ of dimension $r$ with associated $r$-tuple $\left(l_{1}, \ldots, l_{r}\right)$. Then

$$
|\operatorname{supp}(U)| \geq a\left(l_{1}, \ldots, l_{r}\right) .
$$

Proof. Let $U$ be a linear subspace of $C(l)$ of dimension $r$. Let $\mathbf{u}_{1}, \ldots, \mathbf{u}_{r}$ be a basis of $U$ with indices $l \leq l_{1}<\cdots<l_{r} \leq N$ such that $\mathbf{u}_{i} \in C\left(l_{i}\right)$ and $\mathbf{u}_{i} \notin C\left(l_{i}+1\right)$ for all $i=1, \ldots, r$.

Let $M_{k}$ be the $a\left(l_{k}\right) \times N$ submatrix of $S\left(\mathbf{u}_{k}\right)$ with the $a\left(l_{k}\right)$ rows corresponding to the indices of $A\left(l_{k}\right)$. The matrix $M_{k}$ has rank $a\left(l_{k}\right)$, since it has an $a\left(l_{k}\right) \times a\left(l_{k}\right)$ submatrix with nonzeros on the backdiagonal and zeros above the backdiagonal, according to Lemma 3.11. 
Let $M$ be the $\sum a\left(l_{k}\right) \times N$ matrix defined by

$$
M=\left(\begin{array}{c}
M_{1} \\
\vdots \\
M_{r}
\end{array}\right) .
$$

The rank of $M$ is a lower bound of the dimension of $\operatorname{Im}^{*}(S, U)$.

Apply Gaussian elimination to $M$ with the $a\left(l_{1}\right)$ pivots in the rows of $M_{1}$ and the columns corresponding to $A\left(l_{1}\right)$. Suppose that we have found a matrix with pivots in the rows of $M_{1}, \ldots, M_{k}$ and the columns corresponding to $\cup_{i=1}^{k} A\left(l_{i}\right)$. Apply Gaussian elimination with the pivots in the rows of $M_{k+1}$ and the columns corresponding to

$$
A\left(l_{k+1}\right) \backslash\left(\cup_{i=1}^{k} A\left(l_{i}\right)\right) .
$$

So with induction and Gaussian elimination a matrix $M^{\prime}$ is obtained from $M$ with pivots in the columns corresponding with $\cup_{k=1}^{r} A\left(l_{k}\right)$. So $M$, and therefore $\operatorname{Im}^{*}(S, U)$, has rank at least $a\left(l_{1}, \ldots, l_{r}\right)$.

The proof is completed with Lemma 3.5.

Definition 3.13 Let $d_{O R D, \varphi, r}(l)$ be the minimal $a\left(l_{1}, \ldots, l_{r}\right)$ such that $l \leq$ $l_{1}<\cdots<l_{r} \leq N$ and $C\left(l_{i}\right) \neq C\left(l_{i}+1\right)$ for all $i=1, \ldots, r$. It is called the order bound on the $r$ th generalized Hamming weight and will be abbreviated by $d_{r}(l)$.

Theorem 3.14

$$
d_{r}(C(l)) \geq d_{r}(l) .
$$

Proof. This is a direct consequence of Proposition 3.12, the definition of GHW's and Definition 3.13.

Remark 3.15 The codes $C(l)$ can be decoded by means of the scheme of majority voting of unknown syndromes which corrects $\left\lfloor\left(d_{1}(l)-1\right) / 2\right\rfloor$ errors. See $[10,17,30]$. 


\section{Reed-Muller codes}

The theory of the previous section will be applied to RM codes.

Order the monomials by the graded lexicographic order. Let $\mathcal{P}$ be the set of all distinct points of $\mathbb{F}_{q}^{m}$. So, $n=q^{m}$ and $\mathcal{P}=\left\{P_{1}, \ldots, P_{n}\right\}$. Let $\varphi(f)=\left(f\left(P_{1}\right), \ldots, f\left(P_{n}\right)\right)$. The map $\varphi$ is a surjective morphism of $\mathbb{F}_{q^{-}}$ algebras by $[7,16]$. Define $\mathbf{h}_{i}=\varphi\left(f_{i}\right)$ and $C(l)=\left\{\mathbf{c} \in \mathbb{F}_{q}^{n}: \mathbf{c} \cdot \mathbf{h}_{i}=0\right.$ for all $i \leq l\}$.

Since $\varphi\left(f^{q}\right)=\varphi(f)$ many of the parity checks $\mathbf{h}_{i}$ will be the same and therefore adding an extra parity check will not always lead to a different code. A monomial $X^{\alpha}$ is called reduced if $0 \leq \alpha_{j} \leq q-1$ for all $j, 1 \leq j \leq m$. There are exactly $q^{m}=n$ of these monomials. The set $\left\{\varphi\left(X^{\alpha}\right): X^{\alpha}\right.$ is reduced $\}$ is a basis for $\mathbb{F}_{q}^{n}$. A polynomial is called reduced if it is a linear combination of reduced monomials. For every polynomial $f$ there exists a unique reduced polynomial $\bar{f}$ such that $\varphi(f)=\varphi(\bar{f})$.

Lemma 4.1 Let $f \in \mathbb{F}_{q}\left[X_{1}, \ldots, X_{m}\right]$ be reduced. Then

$$
\sum_{i=1}^{n} f\left(P_{i}\right)=\left\{\begin{array}{cll}
0 & \text { if } & \operatorname{deg}(f)<(q-1) m \\
n o t \text { zero } & \text { if } & \operatorname{deg}(f)=(q-1) m
\end{array}\right.
$$

Proof. The proof can be found in $[7,16]$.

Consider the sequence of codes $(C(l): l \in \mathbb{N})$. Then $C(l+1) \subseteq C(l)$, and $\operatorname{dim}(C(l+1)) \geq \operatorname{dim}(C(l))-1$ and $C(l)=0$ for all $l>N$. So $\{C(l): l \in \mathbb{N}\}$ consists of $n$ distinct codes. Define a new sequence $\left(C_{r}\right)_{r=1}^{n}$ such that $C_{r}$ has dimension $n-r$ and $C_{r}=C(l) \neq C(l-1)$ for some $l$.

\section{Proposition 4.2}

$$
C_{r}^{\perp}=C_{n-r}
$$

Proof. Consider the subsequence of the infinite sequence of monomials $\left(f_{i}: i \in \mathbb{N}\right)$ consisting of all reduced monomials $X^{\alpha}$. This new sequence is also ordered by the graded lexicographic order. Let $g_{i}, 1 \leq i \leq n$, be the $i$-th monomial within this new sequence. Then the code $C_{r}$ is, by definition, equal to

$$
C_{r}=\left\{\mathbf{c} \in \mathbb{F}_{q}^{n}: \mathbf{c} \cdot \varphi\left(g_{i}\right)=0 \text { for all } i \leq r\right\}
$$


Therefore $\varphi\left(g_{i}\right)$ is an element of the dual of the code $C_{r}$. We have to prove that $\varphi\left(g_{i}\right)$ is in $C_{n-r}$ for all $i \leq r$. Since

$$
C_{n-r}=\left\{\mathbf{c} \in \mathbb{F}_{q}^{n}: \mathbf{c} \cdot \varphi\left(g_{j}\right)=0 \text { for all } j \leq n-r\right\}
$$

this is the same as to prove that

$$
\varphi\left(g_{i}\right) \cdot \varphi\left(g_{j}\right)=0
$$

for all $i \leq r$ and all $j \leq n-r$. Now

$$
\varphi\left(g_{i}\right) \cdot \varphi\left(g_{j}\right)=\sum_{l=1}^{n}\left(g_{i} g_{j}\right)\left(P_{l}\right)
$$

and Lemma 4.1 implies that this equals zero if the degree of $g_{i} g_{j}$ is smaller than $m(q-1)$.

Let $g_{n}=X^{\mu}=X_{1}^{q-1} \cdots X_{m}^{q-1}$. If $X^{\alpha} \prec X^{\beta}$, then $X^{\mu-\beta} \prec X^{\mu-\alpha}$ since the graded lexicographic order is a reduction order. So the sequence $g_{1}, g_{2}, \ldots, g_{n}$ transforms into the sequence $g_{n}, g_{n-1}, \ldots, g_{1}$ under the operation $X^{\alpha} \mapsto X^{\mu-\alpha}$ and hence $g_{i} g_{j}=g_{n}$ if $i+j=n+1$.

If $i \leq r$ and $j \leq n-r$ then $i+j \leq n$. Let $i^{\prime}=n+1-j$. Then $i<i^{\prime}$, so $g_{i} g_{j}<g_{i^{\prime}} g_{j}=g_{n}$ and $\operatorname{deg}\left(g_{i} g_{j}\right)<\operatorname{deg}\left(g_{n}\right)=m(q-1)$.

Definition 4.3 Let $Q=\{0,1, \ldots, q-1\}$. Let $\mu=(q-1, \ldots, q-1)$. Define $\alpha^{\perp}=\mu-\alpha$ for $\alpha \in Q^{m}$. Let $f_{l}=X^{\alpha}$ be reduced. Define $f_{l}^{\perp}=X^{\alpha^{\perp}}$.

Remark 4.4 The mapping on $Q^{m}$ which sends $\alpha$ to $\alpha^{\perp}$ is a bijection which reverses the lexicographic order.

Corollary 4.5 Let $f \in \mathbb{F}_{q}\left[X_{1}, \ldots, X_{m}\right]$ be reduced. If $f_{l+1}^{\perp}$ is the leading monomial of $f$, then $\varphi(f) \in C(l) \backslash C(l+1)$.

Proof. This a consequence of Proposition 4.2.

Definition 4.6 The $q$-ary Reed-Muller code of order $u$ and in $m$ variables is defined by

$$
\mathcal{R M}_{q}(u, m)=\{\varphi(f): f \in R, \operatorname{deg}(f) \leq u\}
$$


Remark 4.7 Define $u^{\perp}=(q-1) m-u-1$. The dual of the code $\mathcal{R} \mathcal{M}_{q}(u, m)$ is $\mathcal{R} \mathcal{M}_{q}\left(u^{\perp}, m\right)$ by Proposition 4.2. See also [7]. So

$$
\mathcal{R} \mathcal{M}_{q}(u, m)=\left\{\mathbf{c} \in \mathbb{F}_{q}^{n}: \mathbf{c} \cdot \mathbf{h}=0 \text { for } \mathbf{h}=\varphi(f) \text { and } \operatorname{deg}(f) \leq u^{\perp}\right\} .
$$

Let $l$ be the integer such that $f_{l}=X_{1}^{u^{\perp}}$. Then $f_{l+1}=X_{m}^{u^{\perp}+1}$ and $C(l)=$ $\mathcal{R} \mathcal{M}_{q}(u, m)$, since the monomials are ordered by the graded lexicographic order. It will be shown that there exists a subspace $U$ of $\mathcal{R} \mathcal{M}_{q}(u, m)$ of dimension $r$ such that the support of $U$ reaches the bound $d_{r}(l)$.

Definition 4.8 Let $Q=\{0, \ldots, q-1\}$. Enumerate the elements of $\mathbb{F}_{q}$ by $Q$. So $\mathbb{F}_{q}=\left\{a_{0}, \ldots, a_{q-1}\right\}$. If $q$ is prime, then we take for $a_{i}$ the residue of $i$ modulo $q$. Let $\alpha \in Q^{m}$. Then $P_{\alpha}$ is defined by $P_{\alpha}=\left(a_{\alpha_{1}}, \ldots, a_{\alpha_{m}}\right)$.

Remark 4.9 From now on the $n=q^{m}$ points $P_{1}, \ldots, P_{n}$ of $\mathbb{F}_{q}^{m}$ are ordered by taking the $P_{\alpha}$ with the lexicographic order on the $\alpha \in Q^{m}$. In this way we have identified the set $\{1, \ldots, n\}$ with $Q^{m}$. Thus supports of codes in $\mathbb{F}_{q}^{n}$ will be subsets of $Q^{m}$.

Definition 4.10 Let $\alpha \in Q^{m}$. Define the polynomial $f_{\alpha}$ by

$$
f_{\alpha}=\prod_{s=1}^{m} \prod_{\alpha_{s}<t \leq q-1}\left(X_{s}-a_{t}\right)
$$

and the corresponding word $\mathbf{h}_{\alpha}=\varphi\left(f_{\alpha}\right)$.

Remark 4.11 For the degree of $f_{\alpha}$ we find

$$
\operatorname{deg}\left(f_{\alpha}\right)=\sum_{s=1}^{m}\left(q-1-\alpha_{s}\right)=(q-1) m-\sum_{s=1}^{m} \alpha_{s}=(q-1) m-\operatorname{deg}\left(X^{\alpha}\right) .
$$

So, if the degree of the monomial $X^{\alpha}$ is greater than $u^{\perp}$, then the degree of $f_{\alpha}$ is smaller than or equal to $u$ and therefore $\mathbf{h}_{\alpha}$ is a codeword of $\mathcal{R} \mathcal{M}_{q}(u, m)$.

Definition 4.12 The partial order $\preceq_{P}$ on $Q^{m}$ is defined by $\alpha \preceq_{P} \beta$ if and only if $\alpha_{s} \leq \beta_{s}$ for all $s=1, \ldots, m$. Define the downward shadow or the ideal generated by an element $\alpha \in Q^{m}$ by

$$
\Delta \alpha=\left\{\beta \in Q^{m}: \beta \preceq_{P} \alpha\right\} .
$$


Define the ideal generated by a subset $A \subseteq Q^{m}$ by

$$
\Delta A=\bigcup_{\alpha \in A} \Delta \alpha
$$

Define the upward shadow of $\alpha \in Q^{m}$ by

$$
\nabla \alpha=\left\{\beta \in Q^{m}: \alpha \preceq_{P} \beta\right\}
$$

Lemma 4.13 Let $\alpha \in Q^{m}$ such that $f_{l+1}=X^{\alpha}$. Then there is a bijection between $A(l)$ and $\Delta \alpha=\operatorname{supp}\left(\mathbf{h}_{\alpha}\right)$. The number of elements of $A(l)$ is $\prod_{s=1}^{m}\left(\alpha_{s}+1\right)=w t\left(\mathbf{h}_{\alpha}\right)$.

Proof. The set $N(l)$ exists of all pairs $(i, j)$ such that $f_{i} f_{j}=f_{l+1}$, The integer $i$, with $f_{i}=X^{\beta}$, is an element of the set $A(l)$ if and only if $\beta \preceq_{P} \alpha$. So there is a bijection between $A(l)$ and $\Delta \alpha$. Hence $|A(l)|=|\Delta \alpha|=\prod_{s=1}^{m}\left(\alpha_{s}+\right.$ 1).

If $\beta \in Q^{m}$, then

$$
f_{\alpha}\left(P_{\beta}\right) \neq 0 \text { if and only if } \beta \preceq_{P} \alpha .
$$

Hence $\Delta \alpha=\operatorname{supp}\left(\mathbf{h}_{\alpha}\right)$.

Lemma 4.14 Let $l$ be a positive integer. Let $l \leq l_{1}<\cdots<l_{r}$ such that $C\left(l_{i}\right) \neq C\left(l_{i}+1\right)$. Then there exists a subspace $U$ of $C(l)$ of dimension $r$, such that $\left(l_{1}, \ldots, l_{r}\right)$ is its associated $r$-tuple and it has effective length $a\left(l_{1}, \ldots, l_{r}\right)$.

Proof. Define $\alpha(i)$, for $1 \leq i \leq r$, by $f_{l_{i}+1}=X^{\alpha(i)}$. Then $f_{l_{i}+1}^{\perp}$ is the leading monomial of $f_{\alpha(i)}$. Hence

$$
\mathbf{h}_{\alpha(i)}=\varphi\left(f_{\alpha(i)}\right) \in C\left(l_{i}\right) \backslash C\left(l_{i}+1\right),
$$

by Corollary 4.5. Let $U$ be the space generated by $\mathbf{h}_{\alpha(1)}, \ldots, \mathbf{h}_{\alpha(r)}$, then the dimension of $U$ is $r$ and its associated $r$-tuple is $\left(l_{1}, \ldots, l_{r}\right)$.

Furthermore

$$
\operatorname{supp}(U)=\cup_{i=1}^{r} \operatorname{supp}\left(h_{\alpha(i)}\right)=\cup_{i=1}^{r} \Delta \alpha(i)
$$

by Lemma 4.13. There is a bijection between $A\left(l_{1}, \ldots, l_{r}\right)=\cup_{i=1}^{r} A\left(l_{i}\right)$ and $\cup_{i=1}^{r} \Delta \alpha(i)$ by Lemma 4.13. Therefore the effective length of $U$ is $a\left(l_{1}, \ldots, l_{r}\right)$. 
Remark 4.15 The subcode $U$ of Lemma 4.14 depends on the choice of the order of the $q^{m}$ points of $\mathbb{F}_{q}^{m}$. Any permutation $\sigma$ of $\{1, \ldots, m\}$ and any permutation $\tau$ of $Q$ induce a permutation of $Q^{m}$ and give another subcode $U^{\prime}$ of the same code $C(l)$ with the same associated $r$-tuple and effective length.

Proposition 4.16 Let $u^{\perp}=(q-1) m-u-1$. Let $l$ be the positive integer such that $f_{l}=X_{1}^{u^{\perp}}$. Then $C(l)=\mathcal{R} \mathcal{M}_{q}(u, m)$ and $d_{r}(C(l))=d_{r}(l)$.

Proof. This follows directly from Lemma 4.14 and Definition 3.13.

Example 4.17 Let $R=\mathbb{F}_{3}\left[X_{1}, X_{2}, X_{3}\right]$. Consider the graded lexicographic order on the monomials in the variables $X_{1}, X_{2}, X_{3}$. Let $f_{i}$ be the $i$ th monomial in this order.

$\begin{array}{llll}f_{1}=1 & f_{19}=X_{1}^{2} X_{2} & f_{37}=X_{2} X_{3}^{4} & f_{55}=X_{1}^{4} X_{2} \\ f_{2}=X_{3} & f_{20}=X_{1}^{3} & f_{38}=X_{2}^{2} X_{3}^{3} & f_{56}=X_{1}^{5} \\ f_{3}=X_{2} & f_{21}=X_{3}^{4} & f_{39}=X_{2}^{3} X_{3}^{2} & f_{57}=X_{3}^{6} \\ f_{4}=X_{1} & f_{22}=X_{2} X_{3}^{3} & f_{40}=X_{2}^{4} X_{3} & f_{58}=X_{1} X_{3}^{5} \\ f_{5}=X_{3}^{2} & f_{23}=X_{2}^{2} X_{3}^{2} & f_{41}=X_{2}^{5} & f_{59}=X_{2}^{2} X_{3}^{4} \\ f_{6}=X_{2} X_{3} & f_{24}=X_{2}^{3} X_{3} & f_{42}=X_{1} X_{3}^{4} & f_{60}=X_{2}^{3} X_{3}^{3} \\ f_{7}=X_{2}^{2} & f_{25}=X_{2}^{4} & f_{43}=X_{1} X_{2} X_{3}^{3} & f_{61}=X_{2}^{4} X_{3}^{2} \\ f_{8}=X_{1} X_{3} & f_{26}=X_{1} X_{3}^{3} & f_{44}=X_{1} X_{2}^{2} X_{3}^{2} & f_{62}=X_{2}^{5} X_{3}^{1} \\ f_{9}=X_{1} X_{2} & f_{27}=X_{1} X_{2} X_{3}^{2} & f_{45}=X_{1} X_{2}^{3} X_{3} & f_{63}=X_{2}^{6} \\ f_{10}=X_{1}^{2} & f_{28}=X_{1} X_{2}^{2} X_{3} & f_{46}=X_{1} X_{2}^{4} & f_{64}=X_{1} X_{3}^{5} \\ f_{11}=X_{3}^{3} & f_{29}=X_{1} X_{2}^{3} & f_{47}=X_{1}^{2} X_{3}^{3} & f_{65}=X_{1} X_{2} X_{3}^{4} \\ f_{12}=X_{2} X_{3}^{2} & f_{30}=X_{1}^{2} X_{3}^{2} & f_{48}=X_{1}^{2} X_{2} X_{3}^{2} & f_{66}=X_{1} X_{2}^{2} X_{3}^{3} \\ f_{13}=X_{2}^{2} X_{3} & f_{31}=X_{1}^{2} X_{2} X_{3} & f_{49}=X_{1}^{2} X_{2}^{2} X_{3} & f_{67}=X_{1} X_{2}^{3} X_{3}^{2} \\ f_{14}=X_{2}^{3} & f_{32}=X_{1}^{2} X_{2}^{2} & f_{50}=X_{1}^{2} X_{2}^{3} & f_{68}=X_{1} X_{2}^{4} X_{3} \\ f_{15}=X_{1} X_{3}^{2} & f_{33}=X_{1}^{3} X_{3} & f_{51}=X_{1}^{3} X_{3}^{2} & f_{69}=X_{1} X_{2}^{5} \\ f_{16}=X_{1} X_{2} X_{3} & f_{34}=X_{1}^{3} X_{2} & f_{52}=X_{1}^{3} X_{2} X_{3} & f_{70}=X_{1}^{2} X_{3}^{4} \\ f_{17}=X_{1} X_{2}^{2} & f_{35}=X_{1}^{4} & f_{53}=X_{1}^{3} X_{2}^{2} & f_{71}=X_{1}^{2} X_{2} X_{3}^{3} \\ f_{18}=X_{1}^{2} X_{3} & f_{36}=X_{3}^{5} & f_{54}=X_{1}^{4} X_{3} & f_{72}=X_{1}^{2} X_{2}^{2} X_{3}^{2}\end{array}$

and so on. Let $\mathcal{P}$ be the set of all 27 distinct points in $\mathbb{F}_{3}^{3}$. Let $\varphi$ be the evaluation map from $R$ to $\mathbb{F}_{3}^{27}$ defined by $\varphi(f)=\left(f\left(P_{1}\right), \ldots, f\left(P_{27}\right)\right)$. The points $P_{1}, \ldots, P_{27}$ ordered in the lexicographic order are tabulated below 
in the columns. In the tables below a word $\left(x_{1}, x_{2}, \ldots, x_{n}\right)$ is denoted by $x_{1} x_{2} \cdots x_{n}$.

$$
\begin{aligned}
& \varphi\left(X_{1}\right)=000000000111111111222222222 \\
& \varphi\left(X_{2}\right)=000111222000111222000111222 \\
& \varphi\left(X_{3}\right)=012012012012012012012012012
\end{aligned}
$$

The word $\mathbf{h}_{i}$ is defined by $\mathbf{h}_{i}=\varphi\left(f_{i}\right)$ for all $i \in \mathbb{N}$. The codes $C(l)$ are defined by

$$
C(l)=\left\{\mathbf{c} \in \mathbb{F}_{3}^{27}: \mathbf{c} \cdot \mathbf{h}_{i}=0 \text { for all } i \leq l\right\} .
$$

Then $C(l)=0$ for all $l>72$. Consider for instance the code

$$
C(10)=\mathcal{R M}_{3}(2,3)^{\perp}=\mathcal{R} \mathcal{M}_{3}(3,3) .
$$

Let $U$ be a subspace of the code $C(10)$ of dimension 4 , for instance

$$
U=\left\langle\mathbf{h}_{5}, \mathbf{h}_{6}, \mathbf{h}_{13}, \mathbf{h}_{19}\right\rangle .
$$

Then $f_{19}^{\perp}=f_{12}, f_{13}^{\perp}=f_{18}, f_{6}^{\perp}=f_{31}$ and $f_{5}^{\perp}=f_{32}$. So the unique 4-tuple associated with $U$ is $(11,17,30,31)$ by Corollary 4.5 .

The sets $A(11), A(17), A(30)$ and $A(31)$ will be computed.

- $f_{12}=X_{3}^{2} X_{2}$ which can be factorized in

$$
1 \cdot X_{2} X_{3}^{2}=X_{2} \cdot X_{3}^{2}=X_{2} X_{3} \cdot X_{3}
$$

so the set

$$
A(11)=\{1,2,3,5,6,12\} .
$$

- $f_{18}=X_{3} X_{1}^{2}$ which can be factorized in

$$
1 \cdot X_{1}^{2} X_{3}=X_{1} \cdot X_{1} X_{3}=X_{1}^{2} \cdot X_{3},
$$

so the set

$$
A(17)=\{1,2,4,8,10,18\} .
$$

- $f_{31}=X_{3} X_{2} X_{1}^{2}$ which can be factorized in

$$
\begin{gathered}
1 \cdot X_{1}^{2} X_{2} X_{3}=X_{1} \cdot X_{1} X_{2} X_{3}=X_{1}^{2} X_{3} \cdot X_{2}= \\
X_{1}^{2} X_{2} \cdot X_{3}=X_{1}^{2} \cdot X_{2} X_{3}=X_{1} X_{2} \cdot X_{1} X_{3},
\end{gathered}
$$

so the set

$$
A(30)=\{1,2,3,4,6,8,9,10,16,18,19,31\} .
$$


- $f_{32}=X_{2}^{2} X_{1}^{2}$ which can be factorized in

$$
1 \cdot X_{1}^{2} X_{2}^{2}=X_{1} \cdot X_{1} X_{2}^{2}=X_{2} \cdot X_{1}^{2} X_{2}=X_{1}^{2} \cdot X_{2}^{2}=X_{1} X_{2} \cdot X_{1} X_{2}
$$

so the set

$$
A(31)=\{1,3,4,7,9,10,17,19,32\}
$$

Hence $A(11) \cup A(17) \cup A(30) \cup A(31)$ is equal to

$$
\{1,2,3,4,5,6,7,8,9,10,12,16,17,18,19,31,32\}
$$

and the number of elements is

$$
a(11,17,30,31)=17 .
$$

Consider the basis of $U$.

$$
\begin{aligned}
\mathbf{h}_{5}=\varphi\left(X_{3}^{2}\right) & =011011011011011011011011011 \\
\mathbf{h}_{6}=\varphi\left(X_{2} X_{3}\right) & =000012021000012021000012021 \\
\mathbf{h}_{13}=\varphi\left(X_{2}^{2} X_{3}\right) & =000012012000012012000012012 \\
\mathbf{h}_{19}=\varphi\left(X_{1}^{2} X_{2}\right) & =000000000000111222000111222
\end{aligned}
$$

The value $a(11,17,30,31)=17$ is smaller than 22 , the effective length of $U$. In Example 5.14 it will be shown that the minimal value $d_{4}(10)$ will be attained for $a(11,12,14,22)=12$.

Example 4.18 Consider the code $C(20)=\mathcal{R M}_{3}(3,3)^{\perp}=\mathcal{R} \mathcal{M}_{3}(2,3)$. Take

$$
\begin{aligned}
& \alpha(1)=(1,2,1) \\
& \alpha(2)=(2,0,2) \\
& \alpha(3)=(2,2,0) \\
& \alpha(4)=(2,1,2)
\end{aligned}
$$

Since $q=3$ is prime, the elements of $\mathbb{F}_{q}$ can be represented by $\{0,1,2\}$. So

$$
\begin{array}{ll}
f_{\alpha(1)}=\left(X_{1}-2\right)\left(X_{3}-2\right) & =X_{1} X_{3}+X_{1}+X_{3}+1 \\
f_{\alpha(2)}=\left(X_{2}-1\right)\left(X_{2}-2\right) & =X_{2}^{2}+2 \\
f_{\alpha(3)}=\left(X_{3}-1\right)\left(X_{3}-2\right) & =X_{3}^{2}+2 \\
f_{\alpha(4)}=X_{2}-2 & =X_{2}+1
\end{array}
$$

Since the sum of the entries of the $\alpha(i)$ is greater than 3 , the $\mathbf{h}_{\alpha(i)}$ is an element of $\mathcal{R M}_{3}(2,3)$, for all $i, 1 \leq i \leq 4$. So, $U=\left\langle\mathbf{h}_{\alpha(1)}, \mathbf{h}_{\alpha(2)}, \mathbf{h}_{\alpha(3)}, \mathbf{h}_{\alpha(4)}\right\rangle$ is a subspace of dimension 4 of $\mathcal{R M}_{3}(2,3)$. 
First the number of elements in the support of $U$ will be computed.

$$
\begin{array}{llcl}
\mathbf{h}_{\alpha(1)}= & \varphi\left(X_{1} X_{3}+X_{1}+X_{3}+1\right) & = & 120120120210210210000000000 \\
\mathbf{h}_{\alpha(2)} & \varphi\left(X_{2}^{2}+2\right) & = & 222000000222000000222000000 \\
\mathbf{h}_{\alpha(3)} & \varphi\left(X_{3}^{2}+2\right) & = & 200200200200200200200200200 \\
\mathbf{h}_{\alpha(4)}= & \varphi\left(X_{2}+1\right) & = & 111222000111222000111222000
\end{array}
$$

The effective length of $U$ is 23 .

The leading monomials of $f_{\alpha(1)}, f_{\alpha(2)}, f_{\alpha(3)}$, and $f_{\alpha(4)}$ are $f_{28}^{\perp}, f_{30}^{\perp}, f_{32}^{\perp}$ and $f_{48}^{\perp}$, respectively. So the unique 4 -tuple associated with $U$ is $(27,29,31,47)$ by Lemma 4.14 .

Next the sets $A(27), A(29), A(31)$ and $A(47)$ will be calculated.

$$
\begin{aligned}
& A(27)=\{1,2,3,4,6,7,8,9,13,16,17,28\} \\
& A(29)=\{1,2,4,5,8,10,15,18,30\} \\
& A(31)=\{1,3,4,7,9,10,17,19,32\} \\
& A(47)=\{1,2,3,4,5,6,8,9,10,12,15,16,18,19,27,30,31,48\}
\end{aligned}
$$

So $A(27) \cup A(29) \cup A(31) \cup A(47)$ is equal to

$$
\{1,2,3,4,5,6,7,8,9,10,12,13,15,16,17,18,19,27,28,30,31,32,48\}
$$

and therefore $a(27,29,31,47)=23$ which equals the effective length of $U$. In Example 5.13 it will be shown that the minimal value $d_{4}(20)$ will be attained for $a(22,26,27,43)=18$.

\section{Some combinatorics}

It seems that we have translated the problem of the computation of the GHW's of RM codes in an equally difficult question. The aim of this section is to give an explicit description of an $r$-tuple that gives the minimum $a\left(l_{1}, \ldots, l_{r}\right)$ in Definition 3.13 of the order bound $d_{r}(l)$ with the help of results from extremal poset theory.

Remark 5.1 Let $Q=\{0, \ldots, q-1\}$. Consider the set $Q^{m}$ with the lexicographic order $\prec_{L}$. Define $\bar{\alpha}=\left(\alpha_{m}, \ldots, \alpha_{1}\right)$ if $\alpha=\left(\alpha_{1}, \ldots, \alpha_{m}\right)$. Let $\mu=(q-1, \ldots, q-1)$. Then the mapping from $Q^{m}$ to itself defined by $\alpha \mapsto \mu-\alpha$ is a bijection. The antilexicographic order $\prec_{A}$ on $Q^{m}$ is defined by $\alpha \prec_{A} \beta$ if and only if there exists a $t$ such that $\alpha_{i}=\beta_{i}$ for all $i>t$ 
and $\alpha_{t}>\beta_{t}$. Hence $\alpha \prec_{A} \beta$ if and only if $\mu-\bar{\alpha} \prec_{L} \mu-\bar{\beta}$. So apart from the duality $\alpha \mapsto \alpha^{\perp}$ discussed in Remark 4.4, there is a second duality $\alpha \mapsto \bar{\alpha}^{\perp}=\mu-\bar{\alpha}$ on $Q^{m}$ which is an isomorphism of linear orders between $\left(Q^{m}, \prec_{L}\right)$ and $\left(Q^{m}, \prec_{A}\right)$, and which induces an isomorphism between $\Delta \alpha$ and $\nabla \bar{\alpha}^{\perp}$. Everything which will be said about the lexicographic order can equally well be said dually for the antilexicographic order, as done in [41] and the next section.

Recall the definitions from 4.12. The partial order $\preceq_{P}$ on $Q^{m}$ is defined by $\alpha \preceq_{P} \beta$ if and only if $\alpha_{i} \leq \beta_{i}$ for all $i, i=1, \ldots, m$. The ideal generated by element $\alpha \in Q^{m}$ is defined by $\Delta \alpha=\left\{\beta \in Q^{m}: \beta \preceq_{P} \alpha\right\}$. The ideal generated by a subset $A \subseteq Q^{m}$ is defined by $\Delta A=\bigcup_{\alpha \in A} \Delta \alpha$.

Definition 5.2 Let $v$ be a fixed integer, $0 \leq v \leq m(q-1)$. Define the sets $F_{v}$ and $F_{>v}$ by

$$
\begin{aligned}
F_{v} & =\left\{\alpha \in Q^{m}: \operatorname{deg}(\alpha)=v\right\} \\
F_{>v} & =\left\{\alpha \in Q^{m}: \operatorname{deg}(\alpha)>v\right\} .
\end{aligned}
$$

Remark 5.3 The second duality $\alpha \mapsto \bar{\alpha}^{\perp}$ on $Q^{m}$ induces also an isomorphism between $F_{>u^{\perp}}$ with respect to the lexicographic order and the set of all $\alpha \in Q^{m}$ such that $\operatorname{deg}(\alpha) \leq u$ with respect to the antilexicographic order, where $u^{\perp}=m(q-1)-u-1$.

Definition 5.4 The initial segment of length $r$ of a subset of $Q^{m}$ consists of the first $r$ elements of this subset with respect to the lexicographic order. Let $L_{v}(r)$ be the initial segment of length $r$ of $F_{v}$. Let $L_{>v}(r)$ be the initial segment of length $r$ of $F_{>v}$.

Theorem 5.5 Let $A$ be a subset of $F_{v}$ consisting of $r$ elements. Then

$$
\left|\left(\Delta L_{v}(r)\right) \cap F_{v-1}\right| \leq\left|(\Delta A) \cap F_{v-1}\right| .
$$

Proof. This theorem is due to [27] when $Q=\mathbb{N}_{0},[22,26]$ when $Q=\{0,1\}$, and [5] when $Q=\{0, \ldots, q-1\}$.

Corollary 5.6 Let $A$ be a subset of $F_{v}$ consisting of $r$ elements. Then

$$
\left|\Delta L_{v}(r)\right| \leq|\Delta A| \text {. }
$$


Proof. Apply Theorem 5.5 iteratively to each layer $F_{i-1}$ and $(\Delta A) \cap F_{i}$. See the remark after [41, Theorem 6].

Theorem 5.7 Let $A$ be a subset of $F_{>v}$ consisting of $r$ elements. Then

$$
\left|\Delta L_{>v}(r)\right| \leq|\Delta A|
$$

Proof. This theorem is proved in the binary case in [41, Lemma 6] as a consequence of Theorem 5.5 for $q=2$. But since this argument is valid for any $q$ and Theorem 5.5 is true for any $q$, this theorem is also valid for any $q$. See also [2] where this theorem is proved in the more general setting of so called Macaulay posets.

Lemma 5.8 Let $r$ be an integer such that $0 \leq r<q^{m}$. Write

$$
r=\sum_{i=1}^{m} \alpha_{m-i+1} q^{i-1} .
$$

Then $\left(\alpha_{1}, \ldots, \alpha_{m}\right)$ is the $(r+1)$ st element of $Q^{m}$ with respect to the lexicographic order.

Proof. Suppose $\alpha, \beta \in Q^{m}$ and $\alpha \prec_{L} \beta$. Then there exists a $t$ such that $\alpha_{i}=\beta_{i}$ for all $i<t$ and $\alpha_{t}<\beta_{t}$. A straight forward calculation shows that $\sum_{i=1}^{m} \alpha_{m-i+1} q^{i-1}<\sum_{i=1}^{m} \beta_{m-i+1} q^{i-1}$. Consider the map

$$
\sigma: Q^{m} \longrightarrow\left\{0,1, \ldots, q^{m}-1\right\}
$$

defined by $\sigma\left(\alpha_{1}, \ldots, \alpha_{m}\right)=\sum_{i=1}^{m} \alpha_{m-i+1} q^{i-1}$. Then $\sigma$ is well defined. So $\sigma$ is a strictly monotone map between two linear orders with the same number of elements. Hence it is an isomorphism of linear orders.

Proposition 5.9 Let $\alpha$ be the rth element in $F_{>v}$ with respect to the lexicographic order $\prec_{L}$. Then

$$
\Delta L_{>v}(r)=\left\{\beta \in Q^{m}: \beta \prec_{L} \alpha \text { or } \beta=\alpha\right\}
$$

and it has $\sum_{i=1}^{m} \alpha_{m-i+1} q^{i-1}+1$ elements. 
Proof. Define $\preceq_{L}$ by $\beta \preceq_{L} \alpha$ if and only if $\beta \prec_{L} \alpha$ or $\beta=\alpha$. Then the partial order $\preceq_{P}$ is coarser than $\preceq_{L}$, that is to say, if $\beta \preceq_{P} \alpha$, then $\beta \preceq_{L} \alpha$. So $\Delta L_{>v}(r) \subseteq\left\{\beta \in Q^{m}: \beta \preceq_{L} \alpha\right\}$.

If $\beta \preceq_{L} \alpha$, then there exists a $t$ such that $\beta_{i}=\alpha_{i}$ for all $i<t$ and $\beta_{t}<\alpha_{t}$. Define $\gamma$ by $\gamma_{i}=\alpha_{i}$ for all $i<t, \gamma_{t}=\alpha_{t}-1$ and $\gamma_{i}=q-1$ for all $i>t$. Then $\beta \in \Delta \gamma, \gamma \prec_{L} \alpha$ and $\operatorname{deg}(\gamma) \geq \operatorname{deg}(\alpha)-1 \geq v$. If $\operatorname{deg}(\gamma)=v$, then $\alpha_{i}=q-1$ for all $i \geq t$, so $\beta \in \Delta \alpha$. If $\operatorname{deg}(\gamma)>v$, then $\gamma \in L_{>v}(r)$. Hence $\beta \in \Delta L_{>v}(r)$. This proves the converse inclusion. The statement about the number of elements follows from Lemma 5.8.

Theorem 5.10 Let $\alpha$ be the rth element in $Q^{m}$ in the lexicographic order with the property that $\operatorname{deg}(\alpha)>u^{\perp}=(q-1) m-u-1$. Then

$$
d_{r}\left(\mathcal{R} \mathcal{M}_{q}(u, m)\right)=\sum_{i=1}^{m} \alpha_{m-i+1} q^{i-1}+1 .
$$

Proof. Let $v=u^{\perp}$. Let $l$ be the positive integer such that $f_{l}=X_{1}^{v}$. Then $C(l)=\mathcal{R M}_{q}(u, m)$ and $d_{r}(C(l))=d_{r}(l)$ by Proposition 4.16. Let $l \leq l_{1}<\cdots<l_{r}$ such that $C\left(l_{i}\right) \neq C\left(l_{i}+1\right)$ for all $i=1, \ldots, r$. Let $\alpha(i)$ be the exponent of $f_{l_{i}+1}$, so $f_{l_{i}+1}=X^{\alpha(i)}$. Then $\operatorname{deg}(\alpha(i))>v$ and $\operatorname{supp}(U)=\cup_{i=1}^{r} \Delta \alpha(i)$ and the effective length of $U$ is $a\left(l_{1}, \ldots, l_{r}\right)$ by Lemma 4.14. The minimal value of these $a\left(l_{1}, \ldots, l_{r}\right)$ is by definition $d_{r}(l)$ and is obtained for $\left(l_{1}, \ldots, l_{r}\right)$ the $r$-tuple such that $\alpha(1), \ldots, \alpha(r)$ are the first $r$ elements of $F_{>v}$ with respect to the lexicographic order, that is to say the elements of $L_{>v}(r)$ by Theorem 5.7. Moreover if $\alpha(r)=\alpha=\left(\alpha_{1}, \ldots, \alpha_{m}\right)$ is the $r$ th element of $F_{>v}$, then

$$
\left|\cup_{i=1}^{r} \Delta \alpha(i)\right|=\left|\Delta L_{>v}(r)\right|=\sum_{i=1}^{m} \alpha_{m-i+1} q^{i-1}+1 .
$$

Corollary 5.11 For given $m, q$ and $u, 0 \leq u<(q-1) m$. Write $u^{\perp}+1=$ $\theta(q-1)+\mu$ for some $\theta, \mu \in \mathbb{N}_{0}$ with $\mu<q-1$. Then the minimum distance of $\mathcal{R M}_{q}(u, m)$ is equal to $(\mu+1) q^{\theta}$.

Proof. This statement is well-known [7, 18], but it follows also from Theorem 5.10. Let $\alpha_{i}=0$ for all $i<m-\theta, \alpha_{m-\theta}=\mu$, and $\alpha_{i}=q-1$ for all 
$i>m-\theta$. Then $\operatorname{deg}(\alpha)=u^{\perp}+1$. The $m$-tuple $\alpha$ is the first element of $F_{>u^{\perp}}$. Hence

$$
d_{1}\left(\mathcal{R} \mathcal{M}_{q}(u, m)\right)=\sum_{i=1}^{\theta}(q-1) q^{i-1}+\mu q^{\theta}+1=(\mu+1) q^{\theta} .
$$

Corollary 5.12 The Reed-Muller codes satisfy the double chain condition.

Proof. This is proved in the binary case in [20]. Let $d_{r}=d_{r}\left(\mathcal{R} \mathcal{M}_{q}(u, m)\right)$. We have shown in Proposition 5.9 and Theorem 5.10 that $\left\{1,2, \ldots, d_{r}\right\}$ is the support of a subcode of $\mathcal{R M}_{q}(u, m)$ of dimension $r$. So it satisfies the chain condition. Let $\tau(j)=q-1-j$ for all $j \in Q$. Then $\tau$ is a permutation of $Q$. The induced permutation on $Q^{m}$ reverses the lexicographic order on $Q^{m}$, see Remark 4.4. So it transforms the first $t$ coordinates to the last $t$, for any $t$. Hence the last $d_{r}$ coordinates form also the support of a subcode of dimension $r$, by Remark 4.15.

Example 5.13 Consider the code $C(20)=\mathcal{R} \mathcal{M}_{3}(2,3)$. This is a continuation of Example 4.18. Enumerate all the points $P_{1}, \ldots, P_{27}$ of $\mathbb{F}_{3}^{3}$ in lexicographic order.

\begin{tabular}{|cccccccccccccccccc|}
\hline 1 & 2 & 3 & 4 & 5 & 6 & 7 & 8 & $\mathbf{9}$ & 10 & 11 & 12 & 13 & 14 & $\mathbf{1 5}$ & 16 & $\mathbf{1 7}$ & $\mathbf{1 8}$ \\
\hline 0 & 0 & 0 & 0 & 0 & 0 & 0 & 0 & $\mathbf{0}$ & 1 & 1 & 1 & 1 & 1 & $\mathbf{1}$ & 1 & $\mathbf{1}$ & $\mathbf{1}$ \\
0 & 0 & 0 & 1 & 1 & 1 & 2 & 2 & $\mathbf{2}$ & 0 & 0 & 0 & 1 & 1 & $\mathbf{1}$ & 2 & $\mathbf{2}$ & $\mathbf{2}$ \\
0 & 1 & 2 & 0 & 1 & 2 & 0 & 1 & $\mathbf{2}$ & 0 & 1 & 2 & 0 & 1 & $\mathbf{2}$ & 0 & $\mathbf{1}$ & $\mathbf{2}$ \\
\hline
\end{tabular}

\begin{tabular}{|ccccccccc|}
\hline 19 & 20 & $\mathbf{2 1}$ & 22 & $\mathbf{2 3}$ & $\mathbf{2 4}$ & $\mathbf{2 5}$ & $\mathbf{2 6}$ & $\mathbf{2 7}$ \\
\hline 2 & 2 & $\mathbf{2}$ & 2 & $\mathbf{2}$ & $\mathbf{2}$ & $\mathbf{2}$ & $\mathbf{2}$ & $\mathbf{2}$ \\
0 & 0 & $\mathbf{0}$ & 1 & $\mathbf{1}$ & $\mathbf{1}$ & $\mathbf{2}$ & $\mathbf{2}$ & $\mathbf{2}$ \\
0 & 1 & $\mathbf{2}$ & 0 & $\mathbf{1}$ & $\mathbf{2}$ & $\mathbf{0}$ & $\mathbf{1}$ & $\mathbf{2}$ \\
\hline
\end{tabular}

In this enumeration those points for which the sum of their entries is greater than 3 are printed boldface. Their labels in the lexicographic order gives us the generalized weights of $C(20)$.

\begin{tabular}{|c|cccccccccc|}
\hline$r$ & 1 & 2 & 3 & 4 & 5 & 6 & 7 & 8 & 9 & 10 \\
\hline$d_{r}(C)$ & 9 & 15 & 17 & 18 & 21 & 23 & 24 & 25 & 26 & 27 \\
\hline
\end{tabular}


The value for $d_{4}(20)$ is attained for $a(22,26,27,43)=18$, since $f_{23}=X_{2}^{2} X_{3}^{2}$, $f_{27}=X_{1} X_{2} X_{3}^{2}, f_{28}=X_{1} X_{2}^{2} X_{3}$ and $f_{44}=X_{1} X_{2}^{2} X_{3}^{2}$, respectively, corresponding to $(0,2,2),(1,1,2),(1,2,1)$ and $(1,2,2)$, respectively.

Example 5.14 Now consider the code $C(10)=\mathcal{R M}_{3}(3,3)$. This is a continuation of Example 4.17. In the enumeration of points of $\mathbb{F}_{3}^{3}$ we consider now all points for which the sum of their entries is greater than 2 . Their labels in the lexicographic order gives us the generalized weights of $C(10)$.

\begin{tabular}{|c|ccccccccccccccccc|}
\hline$r$ & 1 & 2 & 3 & 4 & 5 & 6 & 7 & 8 & 9 & 10 & 11 & 12 & 13 & 14 & 15 & 16 & 17 \\
\hline$d_{r}(C)$ & 6 & 8 & 9 & 12 & 14 & 15 & 16 & 17 & 18 & 20 & 21 & 22 & 23 & 24 & 25 & 26 & 27 \\
\hline
\end{tabular}

The value for $d_{4}(10)$ is attained by $a(11,12,14,22)$.

\section{A recursive formula}

In this section a recursive formula for the determination of the GHW's of RM codes will be derived.

Definition 6.1 Let $u, v$ and $m$ be nonnegative integers. Define

$$
\begin{aligned}
M_{q}(u, m) & =\left\{X^{\alpha}: \alpha \in Q^{m}, \operatorname{deg}(\alpha) \leq u\right\} \\
M_{q}(u, v, m) & =\left\{X^{\alpha}: \alpha \in Q^{m}, \operatorname{deg}(\alpha) \leq u, \alpha_{m} \leq v\right\} .
\end{aligned}
$$

Definition 6.2 Let $\rho_{q}(u, m)$ and $\rho_{q}(u, v, m)$ denote the number of elements of $M_{q}(u, m)$ and $M_{q}(u, v, m)$, respectively. Most of the time the index $q$ will be deleted.

Remark 6.3 It is clear that $M(u, 0, m)=M(u, m-1)=M(u, q-1, m-1)$ and that $M(u, v, m)=M(u, u, m)$ if $u<v$.

The following lemma is basic to the recursive procedure later in this section.

Lemma 6.4 If $0<v \leq u$, then

$$
\begin{aligned}
M(u, v, m) & =M(u, v-1, m) \cup M(u-v, m-1) X_{m}^{v} \\
\rho(u, v, m) & =\rho(u, v-1, m)+\rho(u-v, m-1) .
\end{aligned}
$$


Proof. If $X^{\alpha} \in M(u, v, m)$, then either $\alpha_{m} \leq v-1$ or $\alpha_{m}=v$. So $M(u, v, m)$ is the disjoint union of $M(u, v-1, m)$ and $M(u-v, m-1) X_{m}^{v}$.

\section{Lemma 6.5}

$$
\rho_{q}(u, m)=\operatorname{dim}\left(\mathcal{R M}_{q}(u, m)\right)=\sum_{i=0}^{u} \sum_{j=0}^{\lfloor i / q\rfloor}(-1)^{j}\left(\begin{array}{c}
m \\
j
\end{array}\right)\left(\begin{array}{c}
m-1+i-q j \\
m-1
\end{array}\right)
$$

Proof. See [35] or [38, Exercise 1.2.8].

Proposition 6.6 Let $u, v$ and $m$ be nonnegative integers such that $v \leq q-1$. Let $r$ be an integer such that $0 \leq r<\rho(u, v, m)$, then $r$ has a canonical representation with respect to $(u, v, m)$, that is to say, there exist a positive integer $t$ and sequences of nonnegative integers $u_{0}, u_{1}, \ldots, u_{t}$ and $m_{0}, m_{1}, \ldots, m_{t}$, and sets $N\left(u_{i}, m_{i}\right)$ of $\rho\left(u_{i}, m_{i}\right)$ monomials such that $\cup_{i=1}^{t} N\left(u_{i}, m_{i}\right)$ is a disjoint union and consists of the first $r$ elements of $M(u, v, m)$ with respect to the antilexicographic order. So

$$
r=\sum_{i=1}^{t} \rho\left(u_{i}, m_{i}\right)
$$

Furthermore

$$
u_{i}=\max \left\{0, u_{i-1}+1-(q-1)\left(m_{i-1}-m_{i}\right)\right\}
$$

for all $i, 1 \leq i \leq t$, where $u_{0}=u+(q-1)-(v+1)$ and $m_{0}=m$. The canonical representation of $r$ with respect to $(u, v, m)$ is unique.

Proof. See [41, Lemma 2] for the binary case and [1] for arbitrary $q$. The idea of the representation is to use by induction Lemma 6.4

$$
M\left(u^{\prime}, v^{\prime}, m^{\prime}\right)=M\left(u^{\prime}, v^{\prime}-1, m^{\prime}\right) \cup M\left(u^{\prime}-v^{\prime}, m^{\prime}-1\right) X_{m^{\prime}}^{v^{\prime}}
$$

All elements of $M\left(u^{\prime}-v^{\prime}, m^{\prime}-1\right) X_{m^{\prime}}^{v^{\prime}}$ are smaller than the elements of $M\left(u^{\prime}, v^{\prime}-1, m^{\prime}\right)$ with respect to the antilexicographic order.

The sequences $\left(u_{i}\right),\left(m_{i}\right)$ and the sets $N\left(u_{i}, m_{i}\right)$ are defined and their properties are proved by induction on the quadruples $(r, u, v, m)$ that are ordered 
with respect to the lexicographic order. If $r=0$, then there is nothing to prove. Assume that the sequences and the sets are defined and have the desired properties for all $r^{\prime}, 0 \leq r^{\prime}<r$.

(i) If $v=0$, then $M(u, v, m)=M(u, q-1, m-1)$ by Remark 6.3. So we may assume $v>0$.

(ii) If $u<v$, then $M(u, v, m)=M(u, u, m)$ by Remark 6.3. So we may assume $v \leq u$.

(iii) Suppose $0<v \leq u$ and $r \geq \rho(u-v, m-1)$. Let $r^{\prime}=r-\rho(u-v, m-1)$. Then $0 \leq r^{\prime}<\rho(u, v-1, m)$, since $\rho(u, v, m)=\rho(u, v-1, m)+\rho(u-v, m-1)$ by Lemma 6.4. By induction $r^{\prime}$ has a canonical representation with respect to $(u, v-1, m)$, so there exists a $t^{\prime}$ and sequences $u_{0}^{\prime}, u_{1}^{\prime}, \ldots, u_{t^{\prime}}^{\prime}$ and $m_{0}^{\prime}$, $m_{1}^{\prime}, \ldots, m_{t^{\prime}}^{\prime}$, and sets $N^{\prime}\left(u_{i}^{\prime}, m_{i}^{\prime}\right)$ consisting of $\rho\left(u_{i}^{\prime}, m_{i}^{\prime}\right)$ elements, with the stated properties. Let $u_{0}=u+(q-1)-(v+1)$ and $m_{0}=m$. Let $u_{1}=u-v$, $m_{1}=m-1$ and $N\left(u_{1}, m_{1}\right)=M\left(u_{1}, m_{1}\right) X_{m}^{v}$. Let $t=t^{\prime}+1$. Let $u_{i}=u_{i-1}^{\prime}$, $m_{i}=m_{i-1}^{\prime}$ and $N\left(u_{i}, m_{i}\right)=N^{\prime}\left(u_{i-1}^{\prime}, m_{i-1}^{\prime}\right)$ for all $2 \leq i \leq t$. In this way a canonical representation of $r$ with respect to $(u, v, m)$ is obtained.

(iv) If $0<v \leq u$ and $0 \leq r<\rho(u-v, m-1)=\rho(u-v, q-1, m-1)$, then by induction $r$ has a canonical representation with respect to $(u-v, q-1, m-1)$. So there exist a positive integer $t^{\prime}$ and sequences of nonnegative integers $u_{0}^{\prime}$, $u_{1}^{\prime}, \ldots, u_{t^{\prime}}^{\prime}$ and $m_{0}^{\prime}, m_{1}^{\prime}, \ldots, m_{t^{\prime}}^{\prime}$, and sets $N^{\prime}\left(u_{i}^{\prime}, m_{i}^{\prime}\right)$ of $\rho\left(u_{i}^{\prime}, m_{i}^{\prime}\right)$ monomials such that $\cup_{i=1}^{t^{\prime}} N^{\prime}\left(u_{i}^{\prime}, m_{i}^{\prime}\right)$ is a disjoint union and consists of the first $r$ elements of $M(u-v, q-1, m-1)$ with respect to the antilexicographic order. Let $t=t^{\prime}$. Let $u_{0}=u+(q-1)-(v+1)$ and $m_{0}=m$. Let $u_{i}=u_{i}^{\prime}$ and $m_{i}=m_{i}^{\prime}$ for all $1 \leq i \leq t$. Let $N\left(u_{i}, m_{i}\right)=N^{\prime}\left(u_{i}, m_{i}^{\prime}\right) X_{m}^{v}$. Hence a canonical representation of $r$ with respect to $(u, v, m)$ is obtained.

The proof of the statement about the $u_{i}$ and of the uniqueness of the canonical representation is left to the reader.

Proposition 6.7 Let $u_{0}, u_{1}, \ldots, u_{t}$ and $m_{0}, m_{1}, \ldots, m_{t}$ be the sequences in the canonical representation of $r$ with respect to $(u, v, m)$. Let

$$
\beta_{i}=u_{i}-\left[u_{i-1}+1-(q-1)\left(m_{i-1}-m_{i}\right)\right]+1,
$$

for $i=1, \ldots, t$. Let $U$ be the code generated by the $\mathbf{h}_{\alpha^{\perp}}$ for the first $r$ elements $X^{\alpha} \in M(u, v, m)$ with respect to the antilexicographic order. Let $R=\rho_{q}(u-v, m-1)$. Then

$$
|\operatorname{supp}(U)|= \begin{cases}(q-v) \sum_{i=1}^{t} \beta_{i} q^{m_{i}} & \text { if } \quad r \leq R, \\ (q-v) \beta_{1} q^{m_{1}}+\sum_{i=2}^{t} \beta_{i} q^{m_{i}} & \text { if } \quad r>R .\end{cases}
$$


Proof. See [1] in case $v=q-1$. The general case is treated in the Appendix.

Theorem 6.8 Let the sequence $\left(\beta_{i}\right)$ be associated to the canonical representation of $r$ with respect to $(u, q-1, m)$ as in Proposition 6.7. Then

$$
d_{r}\left(\mathcal{R} \mathcal{M}_{q}(u, m)\right)=\sum_{i=1}^{t} \beta_{i} q^{m_{i}} .
$$

Proof. See [41, Corollary 6] for the binary case and [1] for $q=3$. It is a consequence of Theorem 5.10, Remark 5.1 and Proposition 6.7 with $v=q-1$.

Remark 6.9 The inductive procedure to obtain the sequences $\left(u_{i}\right)$ and $\left(m_{i}\right)$ and the sets $N\left(u_{i}, m_{i}\right)$ in the proof of Proposition 6.6 can be depicted by a labeled directed graph. The nodes consist of quadruples of the form $(r, u, v, m)$ and the leaves are the pairs $\left(u_{i}, m_{i}\right)$.

Suppose that $(r, u, v, m)$ is the quadruple that corresponds to the state in the proof where the first $r$ monomials are looked for in $M(u, v, m)$.

(i) If $v=0$, then a vertical arrow is drawn from $(r, u, 0, m)$ to $(r, u, q-1, m-1)$ as follows:

$$
\begin{gathered}
(r, u, 0, m) \\
\downarrow \\
(r, u, q-1, m-1)
\end{gathered}
$$

(ii) If $u<v$, then a vertical arrow is drawn from $(r, u, v, m)$ to $(r, u, u, m)$ as follows:

$$
\begin{gathered}
(r, u, v, m) \\
\downarrow \\
(r, u, u, m)
\end{gathered}
$$

(iii) If $0<v \leq u$ and $r \geq \rho(u-v, m-1)$, then two diagonal arrows are drawn from $(r, u, v, m)$. The first down to the left to $\left(r^{\prime}, u, v-1, m\right)$ with $r^{\prime}=r-\rho(u-v, m-1)$. The second down to the right to $(u-v, m-1)$ with the label $X_{m}^{v}$ as follows:

$$
\left(r^{\prime}, u, v-1, m\right) \stackrel{\swarrow}{(r, u, v, m)} \searrow_{m}^{X_{m}^{v}}
$$


(iv) If $0<v \leq u$ and $0 \leq r<\rho(u-v, m-1)$, then a diagonal arrow is drawn from $(r, u, v, m)$ down to the right to $(r, u-v, q-1, m-1)$ with the label $X_{m}^{v}$ as follows:

$$
(r, u, v, m) \searrow_{X_{m}^{v}}(r, u-v, q-1, m-1)
$$

The leaves of the tree are pairs of the form $\left(u_{i}, m_{i}\right)$ where the index $i$ increases with the distance to the node at the top. The set $N\left(u_{i}, m_{i}\right)$ is obtained by mutiplying $M\left(u_{i}, m_{i}\right)$ by the monomials that stand as labels of the edges of the unique path from the top node to the leaf $\left(u_{i}, m_{i}\right)$.

Example 6.10 Let $q=4, r=8$ and $u=v=m=3$. Then the tree looks like:

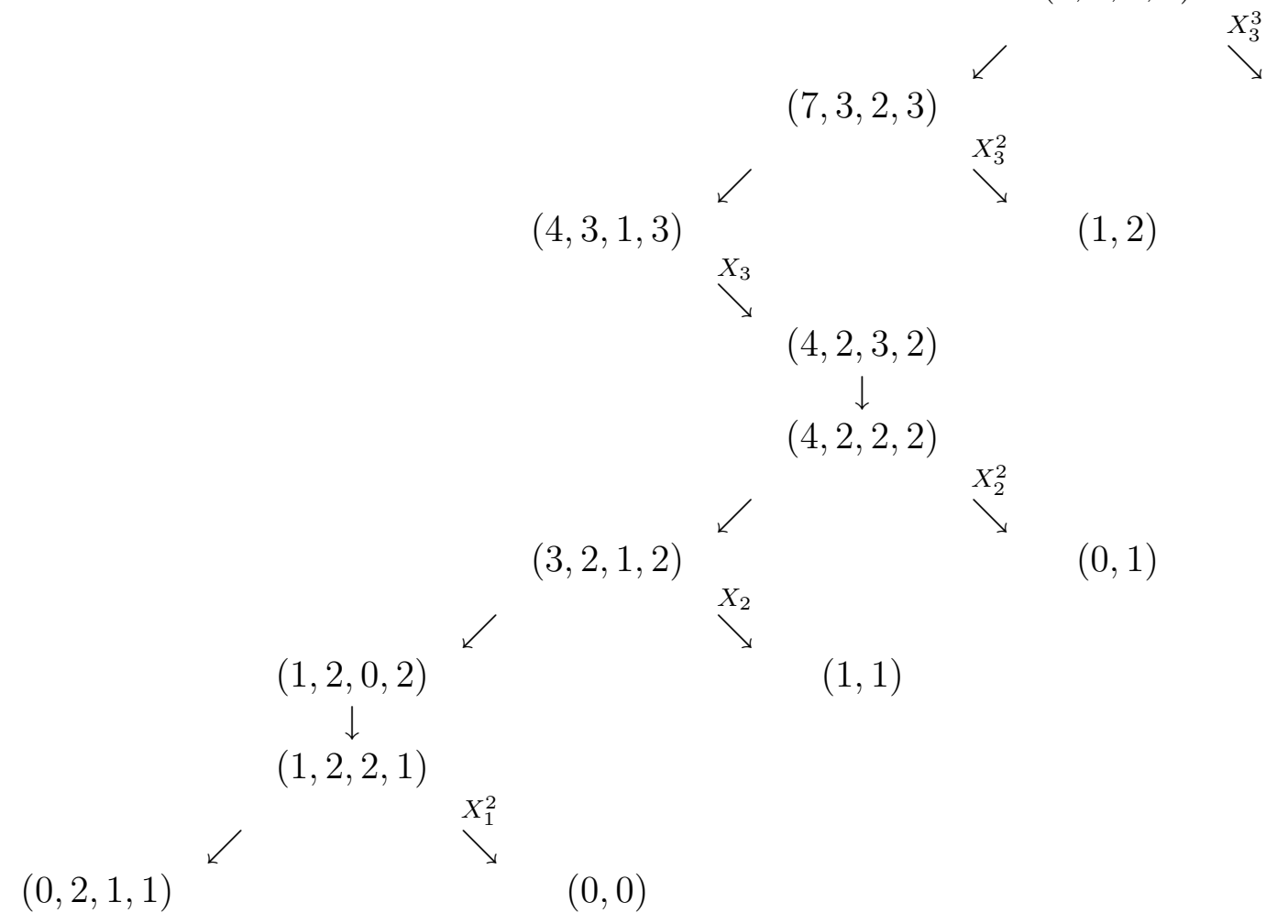

The pairs $\left(u_{i}, m_{i}\right)$, the sets $N\left(u_{i}, m_{i}\right)$ and the $\beta_{i}$ are given in the following 
table.

\begin{tabular}{|c|c|c|c|}
\hline$i$ & $\left(u_{i}, m_{i}\right)$ & $N\left(u_{i}, m_{i}\right)$ & $\beta_{i}$ \\
\hline 0 & $(2,3)$ & & \\
1 & $(0,2)$ & $\{1\} \cdot X_{3}^{3}$ & 1 \\
2 & $(1,2)$ & $\left\{1, X_{1}, X_{2}\right\} \cdot X_{3}^{2}$ & 1 \\
3 & $(0,1)$ & $\{1\} \cdot X_{2}^{2} \cdot X_{3}$ & 2 \\
4 & $(1,1)$ & $\left\{1, X_{1}\right\} \cdot X_{2} \cdot X_{3}$ & 1 \\
5 & $(0,0)$ & $\{1\} \cdot X_{1}^{2} \cdot X_{3}$ & 2 \\
\hline
\end{tabular}

Hence $d_{8}=1 \cdot 4^{2}+1 \cdot 4^{2}+2 \cdot 4^{1}+1 \cdot 4^{1}+2 \cdot 4^{0}=46$ by Theorem 6.8 .

Now $u^{\perp}=(4-1) \cdot 3-3-1=5$. The first 8 elements of $F_{>5}$ are tabulated below.

\begin{tabular}{|l|llllllll|}
\hline$\alpha_{1}$ & 0 & 1 & 1 & 1 & 2 & 2 & 2 & 2 \\
$\alpha_{2}$ & 3 & 2 & 3 & 3 & 1 & 2 & 2 & 3 \\
$\alpha_{3}$ & 3 & 3 & 2 & 3 & 3 & 2 & 3 & 1 \\
\hline
\end{tabular}

Hence $d_{8}=1 \cdot 4^{0}+3 \cdot 4^{1}+2 \cdot 4^{2}+1=46$ by Theorem 5.10 .

Remark 6.11 Notice that in the binary case $v=0$ or $v=1$. The only occurrence of $v=0$ in the tree is directly after case (iii). Hence the index for $v$ is superfluous and can be deleted. Case (iii) gives rise to the diagram:

$$
\left(r^{\prime}, u, m-1\right) \swarrow^{(r, u, m)} \searrow_{(u-1, m-1)}^{X_{m}}
$$

Furthermore $u_{i}=u_{i-1}+1-\left(m_{i-1}-m_{i}\right)$, so $\beta_{i}=1$ for all $i=1, \ldots, t$ when $q=2$.

Example 6.12 Let $q=2, r=10, u=3$, and $m=5$. Then the tree looks 
like:

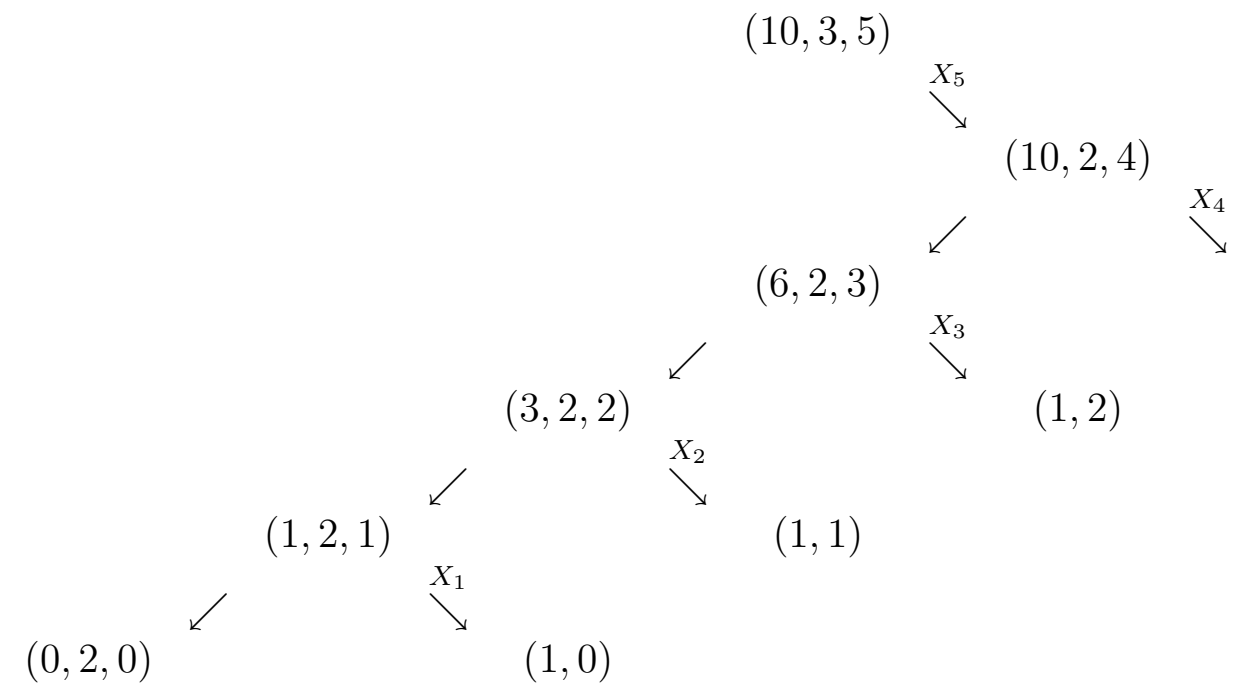

The pairs $\left(u_{i}, m_{i}\right)$ and sets $N\left(u_{i}, m_{i}\right)$ are given in the following table.

\begin{tabular}{|c|c|c|}
\hline$i$ & $\left(u_{i}, m_{i}\right)$ & $N\left(u_{i}, m_{i}\right)$ \\
\hline 0 & $(2,5)$ & \\
1 & $(1,3)$ & $\left\{1, X_{1}, X_{2}, X_{3}\right\} \cdot X_{4} \cdot X_{5}$ \\
2 & $(1,2)$ & $\left\{1, X_{1}, X_{2}\right\} \cdot X_{3} \cdot X_{5}$ \\
3 & $(1,1)$ & $\left\{1, X_{1}\right\} \cdot X_{2} \cdot X_{5}$ \\
4 & $(1,0)$ & $\{1\} \cdot X_{1} \cdot X_{5}$ \\
\hline
\end{tabular}

Hence $d_{10}=2^{3}+2^{2}+2^{1}+2^{0}=15$ by Theorem 6.8 .

Now $u^{\perp}=(2-1) \cdot 5-3-1=1$. The 10 th element of $F_{>1}$ is $(0,1,1,1,0)$. So $d_{10}=0 \cdot 2^{0}+1 \cdot 2^{1}+1 \cdot 2^{2}+1 \cdot 2^{3}+0 \cdot 2^{4}+1=15$ by Theorem 5.10.

The sets $N\left(u_{i}, m_{i}\right)$ are not always of the form $M\left(u_{i}, m_{i}\right) \prod_{j=m_{i}+1}^{m_{i}+u-u_{i}} X_{j}$ as claimed in the proof of [41, Corollary 6].

\section{Concluding remarks and questions}

The above methods readily apply to the case where $\mathcal{P}$ corresponds to a multidimensional block of the form $\left\{\alpha \in Q^{m}: 0 \leq \alpha_{i} \leq q_{i}-1\right.$ for all $\left.i\right\}$, where the $q_{i}$ are positive integers such that $q_{i} \leq q$ for all $i$.

Also the weighted (affine) RM codes [36] can be treated by our methods by considering the weighted graded lexicographic order where the degree of the monomials is weighted. 
Problem 7.1' of [39] is still an open question, that is to say what the GHW's of the projective or nonprimitive RM codes [35, 43] are and whether the above methods also apply to these codes. In [3] and [44] the GHW's of the first and second order projective RM codes are computed. In [3] a conjecture is formulated for the GHW's of projective RM codes which is quite similar in spirit to the answer given in Theorem 5.10 for the ordinary RM codes.

The fundamental problem of the determination of the (generalized) weight distribution [25, 34] of RM codes seems still far out of reach, but Remark 4.15 gives a lot of subcodes with a known effective length.

Let $R$ be an $\mathbb{F}$-algebra. A weight function on $R$ is an order function on $R$ that satisfies furthermore

$$
(O .5) \quad \rho(f g)=\rho(f)+\rho(g)
$$

for all $f, g \in R$. Here $-\infty+n=-\infty$ for all $n \in \mathbb{N}_{0}$.

The standard example of an $\mathbb{F}$-algebra $R$ with a degree function $\rho$ is obtained by taking $R=\mathbb{F}\left[X_{1}, \ldots, X_{m}\right]$ and $\rho(f)=\operatorname{deg}(f)$, the degree of $f \in R$. It is a weight function if and only if $m=1$. More generally, let $\mathcal{X}$ be an algebraic curve defined over the field $\mathbb{F}$. Let $P$ be a rational point of $\mathcal{X}$. Let $R$ be the ring of rational functions on $\mathcal{X}$ which have only poles at the point $P$. Let $v_{P}$ be the discrete valuation at $P$ on $R$. Let $\rho(f)=-v_{P}(f)$, so it is the pole order of $f$ at $P$. Then $\rho$ is a weight function on $R$. See $[16,31,37,38]$.

In $[40,29]$ bounds are given for the GHW's of algebraic geometry codes involving the gonality sequence. Good bounds are obtained [16, 23] for the minimum distance of the one point AG codes in terms of the Weierstrass semigroup of the point at infinity, or what amounts to the same, the semigroup of the weight function $\rho$. This is generalized with the theory of Gröbner bases for affine algebraic sets in [33].

\section{A Proof of Proposition 6.7}

Let $\alpha(1), \ldots, \alpha(r)$ be such that $X^{\alpha(i)}$ is the $i$ th element of $M_{q}(u, v, m)$ with respect to the antilexicographic order, for $i=1, \ldots, r$.

Let $r=1$. The first element $X^{\alpha(1)}$ in $M_{q}(u, v, m)$ with respect to the antilex- 
icographic order is

$$
\alpha(1)=(\underbrace{0, \ldots, 0}_{m-\theta-2}, \mu, \underbrace{q-1, \ldots, q-1}_{\theta}, v),
$$

where $u-v=\theta(q-1)+\mu, \mu<q-1$.

The size of the support of $U$ if $r=1$, is

$$
|\nabla \alpha(1)|=q^{m-\theta-2}(q-\mu)(q-v),
$$

by Lemma 4.13 and Remark 5.1. For the canonical representation of $r=1$, we find $u_{0}=u+(q-1)-(v+1), m_{0}=m, u_{1}=0$ and

$$
m_{1}= \begin{cases}m-1 & \text { if } \quad v=u \\ m-2-\theta & \text { if } \quad v<u .\end{cases}
$$

Then

$$
\beta_{1}=u_{1}-\left[u_{0}+1-(q-1)\left(m_{0}-m_{1}\right)\right]+1=\left\{\begin{array}{lll}
1 & \text { if } & u=v \\
q-\mu & \text { if } & u>v
\end{array}\right.
$$

So

$$
(q-v) \beta_{1} q^{m_{1}}=(q-v)(q-\mu) q^{m-\theta-2}
$$

in both cases $u=v$ and $u>v$, and it is equal to the effective length of $U$. Hence the proposition is proved for $r=1$.

Assume from now on that $r>1$. Let $j+1$ be the first nonzero position of $\alpha(r-1)$. So

$$
\alpha(r-1)=\left(\alpha_{1}, \alpha_{2}, \ldots, \alpha_{m}\right)=(\underbrace{0, \ldots, 0}_{j}, \alpha_{j+1}, \ldots, \alpha_{m}),
$$

with $\alpha_{j+1} \neq 0$.

We want to express $\alpha(r)$ in terms of $\alpha(r-1)$. Four cases 1(a)i, 1(a)ii, 1(b) and 2 will be considered. Later case 2 will be subdivided in $2(a), 2(b) i$ and 2(b)ii. Define

$$
\begin{aligned}
& M=u+1-\sum_{l=j+1}^{m} \alpha_{l}, \\
& F=\left\lfloor\frac{M}{q-1}\right\rfloor .
\end{aligned}
$$


Case 1(a)i If $j>0, r>2$ and $\alpha_{1}(r-2)=1$, and $F<j$, then

$$
\alpha(r)=(\underbrace{0, \ldots, 0}_{j-F-1}, M-F(q-1), \underbrace{q-1, \ldots, q-1}_{F}, \alpha_{j+1}-1, \alpha_{j+2}, \ldots, \alpha_{m}) .
$$

Case 1(a)ii If $j>0, r>2$ and $\alpha_{1}(r-2)=1$, and $F \geq j$, then

$$
\alpha(r)=(\underbrace{q-1, \ldots, q-1}_{j}, \alpha_{j+1}-1, \alpha_{j+2}, \ldots, \alpha_{m}) .
$$

Case $\mathbf{1}(\mathbf{b})$ If $j>0$ and $r=2$ or $\alpha_{1}(r-2) \neq 1$, then

$$
\alpha(r)=(\underbrace{0, \ldots, 0}_{j-1}, 1, \alpha_{j+1}-1, \alpha_{j+2}, \ldots, \alpha_{m}) .
$$

Case 2 If $j=0$, that is to say $\alpha_{1} \neq 0$, then

$$
\alpha(r)=\left(\alpha_{1}-1, \alpha_{2}, \ldots, \alpha_{m}\right) .
$$

In all four cases it is easy to verify that

$$
\alpha(r-1) \prec_{A} \alpha(r),
$$

and that there exists no $\beta$ such that $X^{\beta} \in M_{q}(u, v, m)$ and

$$
\alpha(r-1) \prec_{A} \beta \prec_{A} \alpha(r),
$$

since $\operatorname{deg}(\beta)>u$ otherwise.

Hence $\alpha(r)$ can be determined from $\alpha(r-1)$ and $\alpha(r-2)$.

Let $U(r)=\left\langle\mathbf{h}_{\alpha^{\perp}(1)}, \ldots, \mathbf{h}_{\alpha^{\perp}(r)}\right\rangle$. Let $S U(r)$ be the effective length of $U(r)$. We want to express $S U(r)$ in terms of $S U(r-1)$. Now

$$
S U(r)=\left|\cup_{i=1}^{r} \nabla \alpha(i)\right|
$$

by the dual version of Lemma 4.14 and Remark 5.1. Hence

$$
S U(r)=S U(r-1)+\left|\nabla \alpha(r) \backslash \cup_{i=1}^{r-1} \nabla \alpha(i)\right|
$$

The second term at the right is described by the following lemma. 
Lemma A.1 Let $\gamma=\left(\gamma_{1}, \ldots, \gamma_{m}\right)$, then

$$
\gamma \in \nabla \alpha(r) \backslash \cup_{i=1}^{r-1} \nabla \alpha(i)
$$

if and only if

$$
\begin{cases}\alpha(r-1) \prec_{A}\left(\gamma_{1}, \ldots, \gamma_{m-1}, v\right) \preceq_{A} \alpha(r), \gamma_{m} \leq v, & \text { if } r \leq R \\ \alpha(r-1) \prec_{A} \gamma \preceq_{A} \alpha(r), & \text { if } \quad r>R .\end{cases}
$$

Proof. This lemma is the dual version of Proposition 5.9, see Remark 5.1.

\section{Case 1(a)i}

$$
S U(r)= \begin{cases}S U(r-1)+q^{j-F-1}(q-M+F(q-1))(q-v) & \text { if } r \leq R \\ S U(r-1)+q^{j-F-1}(q-M+F(q-1)) & \text { if } r<R\end{cases}
$$

Proof. Let $\gamma \in \nabla \alpha(r)$, then

$\gamma=\left(\geq 0, \ldots, \geq 0, \geq M-F(q-1), q-1, \ldots, q-1, \geq \alpha_{j+1}-1, \geq \alpha_{j+2}, \ldots, \geq \alpha_{m}\right)$.

And if $\alpha(r-1) \prec_{A} \gamma$, then

$\gamma=\left(\geq 0, \ldots, \geq 0, \geq M-F(q-1), q-1, \ldots, q-1, \alpha_{j+1}-1, \alpha_{j+2}, \ldots, \alpha_{m}\right)$.

Hence by Lemma A.1

$$
\gamma \in \nabla \alpha(r) \backslash \cup_{i=1}^{r-1} \nabla \alpha(i)
$$

and there are

$$
q^{j-F-1}(q-M+F(q-1))(q-v)
$$

of these distinct $\gamma^{\prime}$ s if $r \leq R$, and

$$
q^{j-F-1}(q-M+F(q-1))
$$

if $r>R$.

\section{Case 1(a)ii}

$$
S U(r)= \begin{cases}S U(r-1)+(q-v) & \text { if } r \leq R \\ S U(r-1)+1 & \text { if } r>R\end{cases}
$$

Proof. If

$$
\gamma=\left(q-1, \ldots, q-1, \alpha_{j+1}+1, \alpha_{j+2}, \ldots, \alpha_{m-1}, \alpha_{m}\right),
$$


then $\alpha(r-1) \prec_{A} \gamma \leq_{A} \alpha(r)$ and there are $(q-v)$ of these distinct $\gamma$ 's if $r \leq R$, and 1 if $r>R$.

Case 1(b)

$$
S U(r)= \begin{cases}S U(r-1)+q^{j-1}(q-1)(q-v) & \text { if } r \leq R \\ S U(r-1)+q^{j-1}(q-1) & \text { if } r<R .\end{cases}
$$

Proof. If

$$
\gamma=\left(\geq 0, \ldots, \geq 0, \geq 1, \alpha_{j+1}+1, \alpha_{j+2}, \ldots, \alpha_{m-1}, \alpha_{m}\right),
$$

then $\alpha(r-1) \prec_{A} \gamma \leq_{A} \alpha(r)$ and there are $q^{j-1}(q-1)(q-v)$ of these distinct $\gamma^{\prime}$ 's if $r \leq R$, and $q^{j-1}(q-1)$ if $r>R$.

\section{Case 2}

Proof. If

$$
S U(r)= \begin{cases}S U(r-1)+(q-v) & \text { if } r \leq R \\ S U(r-1)+1 & \text { if } r>R .\end{cases}
$$

$$
\gamma=\left(\alpha_{1}+1, \alpha_{2}, \ldots, \alpha_{m-1}, \alpha_{m}\right),
$$

then $\alpha(r-1) \prec_{A} \gamma \leq_{A} \alpha(r)$ and there are $(q-v)$ of these distinct $\gamma$ 's if $r \leq R$, and 1 if $r>R$.

Let $u(r-1)=\left(u_{0}, \ldots, u_{t}\right)$ and $m(r-1)=\left(m_{0}, \ldots, m_{t}\right)$ be the sequences in the canonical representation of $r-1$. Let $\beta(r-1)=\left(\beta_{1}, \ldots, \beta_{t}\right)$. Let $u(r)=\left(\bar{u}_{1}, \ldots, \bar{u}_{\tau}\right)$ and $m(r)=\left(\bar{m}_{1}, \ldots, \bar{m}_{\tau}\right)$ be the sequences in the canonical representation of $r$.

In the following six cases the $u(r)$ and $m(r)$ are expressed in terms of $u(r-1)$ and $m(r-1)$. The reader is advised to draw the corresponding directed labeled graphs as explained in Remark 6.9.

Note that if $\gamma=\left(0, \ldots, 0, \alpha_{j+2}, \ldots, \alpha_{m}\right)$, then

$$
X^{\alpha(r-1)}=X_{j+1}^{\alpha_{j+1}} \cdot X^{\gamma} .
$$

1. (a) i. In this case

$$
X^{\alpha(r)}=X_{j-F}^{M-F(q-1)} \cdot X_{j-F+1}^{q-1} \cdots X_{j}^{q-1} \cdot X_{j+1}^{\alpha_{j+1}-1} \cdot X^{\gamma} .
$$

$\bar{u}_{i}=u_{i}$ and $\bar{m}_{i}=m_{i}$ for $1 \leq i \leq t$ and $\tau=t+1$. Then the tree to obtain the canonical representation of $r$ is an extension of the tree of the canonical representation of $r-1$.

Hence $m_{t}=j, u_{t}=M-1, \bar{u}_{\tau}=0$ and $\bar{m}_{\tau}=j-F-1$. And

$$
\beta(r)=\left(\beta_{1}, \ldots, \beta_{t}, M-1+(q-1)(F-1)\right) .
$$


ii. Now

$$
X^{\alpha(r)}=X_{1}^{q-1} \cdots X_{j}^{q-1} \cdot X_{j+1}^{\alpha_{j+1}-1} \cdot X^{\gamma}
$$

$\bar{u}_{i}=u_{i}$ and $\bar{m}_{i}=m_{i}$ for $1 \leq i \leq t$ and $\tau=t+1$. Then the tree to obtain the canonical representation of $r$ is an extension of the tree of the canonical representation of $r-1$.

Hence $m_{t}=j, \bar{u}_{\tau}=u_{t}+1-j(q-1)$ and $\bar{m}_{\tau}=0$. And

$$
\beta(r)=\left(\beta_{1}, \ldots, \beta_{t}, 1\right) \text {. }
$$

(b) In this case

$$
X^{\alpha(r)}=X_{j} \cdot X_{j+1}^{\alpha_{j+1}-1} \cdot X^{\gamma} .
$$

$\bar{u}_{i}=u_{i}$ and $\bar{m}_{i}=m_{i}$ for $1 \leq i \leq t$ and $\tau=t+1$. Then the tree to obtain the canonical representation of $r$ is an extension of the tree of the canonical representation of $r-1$.

Hence $u_{t}=0, m_{t}=j, \bar{u}_{\tau}=0$ and $\bar{m}_{\tau}=j-1$. And

$$
\beta(r)=\left(\beta_{1}, \ldots, \beta_{t}, q-1\right) .
$$

2. Now

$$
X^{\alpha(r)}=X^{\alpha_{1}-1} \cdot X^{\gamma}
$$

(a) If $\alpha_{1}>1$, then $\bar{u}_{i}=u_{i}$ and $\bar{m}_{i}=m_{i}$ for $1 \leq i \leq t$ and $\tau=t+1$. Then the tree to obtain the canonical representation of $r$ is an extension of the tree of the canonical representation of $r-1$.

Hence $m_{t}=0, \bar{m}_{\tau}=0$ and $\bar{u}_{\tau}=u_{t}+1$. And

$$
\beta(r)=\left(\beta_{1}, \ldots, \beta_{t}, 1\right) .
$$

(b) If $\alpha_{1}=1$, then let $\alpha(r)=\left(0, \ldots, 0, \alpha_{s}, \ldots, \alpha_{m}\right), \alpha_{s} \neq 0$.

Then

$$
N\left(\bar{u}_{\tau}, \bar{m}_{\tau}\right)=X^{\alpha(r)} \cdot M\left(\bar{u}_{\tau} \bar{m}_{\tau}\right) .
$$

Therefore $\bar{m}_{\tau}=s-1$ and $\bar{u}_{\tau}=u-\sum_{i=s}^{m} \alpha_{i}$ and $\tau$ is defined by

$$
\sum_{i=\tau}^{t} \rho\left(u_{i}, m_{i}\right)+1=\rho\left(\bar{u}_{\tau}, \bar{m}_{\tau}\right) .
$$

Furthermore $\bar{u}_{i}=u_{i}$ and $\bar{m}_{i}=m_{i}$ for $i<\tau$. 
i. If $\alpha_{s} \leq q-2$, then let $\gamma=\left(0, \ldots, 0, \alpha_{s+1}, \ldots, \alpha_{m}\right)$.

Then $X^{\alpha(r)}=X_{s}^{\alpha_{s}} \cdot X^{\gamma}$ and $u_{\tau-1}=u-\sum_{i=s}^{m} \alpha_{i}-1$ and $m_{\tau-1}=s-1$. Hence $\beta(r)=\left(\beta_{1}, \ldots, \beta_{\tau-1}, 1\right)$.

ii. If $\alpha_{s}=q-1$, then let $\gamma=\left(0, \ldots, 0, \alpha_{s+2}, \ldots, \alpha_{m}\right)$.

Then $X^{\alpha(r)}=X_{s}^{q-1} \cdot X^{\gamma}$ and $u_{\tau-1}=u-\sum_{i=s+1}^{m} \alpha_{i}-1$ and $m_{\tau-1}=s$. Hence $\beta(r)=\left(\beta_{1}, \ldots, \beta_{\tau-1}, 1\right)$.

This ends the treatment of the six cases.

Let $r \leq R$ and assume that

$$
S U(r-1)=(q-v) \sum_{i=1}^{t} \beta_{i}(r-1) q^{m_{i}} .
$$

Define $B S(r)$ as

$$
B S(r)=(q-v) \sum_{i=1}^{\tau} \beta_{i}(r) q^{\bar{m}_{i}} .
$$

Hence $B S(r-1)=S U(r-1)$ by the induction hypothesis. It remains to prove that in that case $B S(r)=S U(r)$.

Case 1(a)i

$$
\begin{aligned}
& S U(r)-S U(r-1)=q^{j-F-1}(q-M+F(q-1))(q-v) \\
& B S(r)-B S(r-1)=(q-v)(M-1+(q-1)(F-1)) q^{j-F-1}
\end{aligned}
$$

The other cases are left to the reader.

For $r>R$ the proof is the same, since in $S U(r)$ only the factor $(q-v)$ has disappeared, just as in the Proposition.

\section{References}

[1] A. Ashikhmin, "Generalized Hamming weights of Reed-Muller codes over GF(3)," (in Russian) Chapter of Ph.D. Thesis, Institute for Problems of Information Transmission, Moscow, 1994.

[2] S. Bezrukov and P. Heijnen, "On minimal ideals in Macaulay posets," preprint Feb. 1996. 
[3] M. Boguslavsky, "On the number of solutions of polynomial systems," to appear in Finite Fields and their Applications.

[4] B. Buchberger, "Ein Algorithmus zum Auffinden der Basiselemente des Restklassenringes nach einem nulldimensionalen Polynomideal," Ph.D. Thesis, Univ. of Innsbruck, Austria, 1965.

[5] G.F. Clements and B. Lindström, "A generalization of a combinatorial theorem of Macaulay," Journ. Combinatorial Theory, vol. 7, pp. 230-238, 1969.

[6] D. Cox, J. Little and D. O'Shea, Ideals, varieties, and algorithms: an introduction to computational algebraic geometry, Springer-Verlag, Berlin, 1992.

[7] P. Delsarte, J.M. Goethals and F.J. MacWilliams, "On generalized ReedMuller codes and their relatives," Information and Control, vol. 16, pp. 403-442, 1970.

[8] S.B. Encheva, "On binary linear codes which satisfy the two-way chain condition," IEEE Trans. Inform. Theory, vol. IT-42, pp. 1038-1047, May 1996.

[9] O. Endler, Valuation theory, Universitext, Springer-Verlag, Berlin, 1972

[10] G.-L. Feng and T.R.N. Rao, "Decoding of algebraic geometric codes up to the designed minimum distance," IEEE Trans. Inform. Theory, vol. IT-39, pp. 37-45, Jan. 1993.

[11] G.-L. Feng and T.R.N. Rao, "A simple approach for construction of algebraic-geometric codes from affine plane curves," IEEE Trans. Inform. Theory, vol. IT-40, pp. 1003-1012, July 1994.

[12] G.-L. Feng, V. Wei, T.R.N. Rao and K.K. Tzeng, "Simplified understanding and efficient decoding of a class of algebraic-geometric codes," IEEE Trans. Inform. Theory, vol. IT-40, pp. 981-1002, July 1994.

[13] G.D. Forney Jr., "Dimension/length profiles and trellis complexity of linear block codes," IEEE Trans. Inform. Theory, vol. IT-40, pp. 17411752, Nov. 1994. 
[14] G.D. Forney Jr., "Dimension/length profiles and trellis complexity of lattices," IEEE Trans. Inform. Theory, vol. IT-40, pp. 1753-1772, Nov.1994.

[15] T. Helleseth, T. Kløve and J. Mykkelveit, "The weight distibution of irreducible cyclic codes with block lengths $n_{1}\left(\left(q^{l}-1\right) / N\right)$," Discrete Math., vol. 18, pp. 179-211, 1977.

[16] T. Høholdt, J.H. van Lint and R. Pellikaan, "Algebraic geometry codes", to appear in Handbook of Coding Theory, R.A. Brualdi, W.C. Huffman and V. Pless (Eds.), Elsevier, Amsterdam.

[17] T. Høholdt and R. Pellikaan, "On decoding algebraic-geometric codes," IEEE Trans. Inform. Theory. vol. IT-41, pp. 1589-1614, Nov. 1995.

[18] T. Kasami, S. Lin and W.W. Peterson, "New generalization of ReedMuller codes. Part 1: Primitive codes," IEEE Trans. Inform. Theory, vol. IT-14, pp. 189-199, 1968.

[19] T. Kasami, S. Lin and W.W. Peterson, "Polynomial codes," IEEE Trans. Inform. Theory, vol. IT-14, pp. 807-814, 1968.

[20] T. Kasami, T. Takata, T. Fujiwara and S. Lin, "On the optimum bit orders with respect to the state complexity of trellis diagrams for linear binary codes," IEEE Trans. Inform. Theory, vol. IT-39, pp. 242-245, Jan. 1993.

[21] T. Kasami, T. Takata, T. Fujiwara and S. Lin, "On complexity of trellis structure of linear block codes," IEEE Trans. Inform. Theory, vol. IT-39, pp. 1057-1064, May 1993.

[22] G.O.H. Katona, "A theorem of finite sets," in Theory of Graphs, Proceedings of the Colloquium held at Tihany, Hungary, September 1966, ed. by P. Erdös and G. Katona, Academic Press, New York; Akademia Kiado, Budapest 1968, pp. 187-207.

[23] C. Kirfel and R. Pellikaan, "The minimum distance of codes in an array coming from telescopic semigroups," IEEE Trans. Inform. Theory, vol. IT-41, pp. 1720-1732, Nov. 1995.

[24] T. Kløve, "The weight distribution of linear codes over $G F\left(q^{l}\right)$ having generator matrix over $G F(q)$," Discrete Math., vol. 23, pp. 159-168, 1978. 
[25] T. Kløve, "Support weight distribution of linear codes," Discrete Math., vol. 106/107, pp. 311-316, 1992.

[26] J.B. Kruskal, "The optimal number of simplices in a complex," in Math. Optimization Techniques, Univ. Calif. Press, Berkeley, California, 1963, pp. 251-268.

[27] F.S. Macaulay, "Some properties of enumeration in the theory of modular systems," Proc. London Math. Soc., vol. 26, pp. 531-555, 1927.

[28] R.E. Muller, "Application of Bollean algebra to switching circuit design and to error detection," IRE Trans. Electronic Comput., vol. EC-3, pp. 6-12, 1954.

[29] C. Munuera, "On the generalized Hamming weights of geometric Goppa codes," IEEE Trans. Inform. Theory, vol. IT-40, pp. 2092-2099, Nov. 1994.

[30] R. Pellikaan, "The shift bound for cyclic, Reed-Muller and geometric Goppa codes," in Arithmetic, Gemetry and Coding Theory, ed. by R. Pellikaan, M. Perret and S.G. Vlădut, Walter de Gruyter, Berlin 1996, pp. $155-175$.

[31] R. Pellikaan, "On the existence of order and weight functions," to appear in Journ. Statistical Planning and Inference.

[32] I.S. Reed, "A class of multiple-error-correcting codes and the decoding scheme," IRE Trans. Inform. Theory, vol. PGIT-4, pp. 38-49, 1954.

[33] T. Shibuya, J. Mizutani and K. Sakaniwa, "On generalized Hamming weights of codes constructed on affine algebraic sets," in Proc. of AAECC12, Lect. Notes Comp. Science, vol. 1255, pp. 311-320, 1997.

[34] J. Simonis, "The effective length of subcodes," $A A E C C$, vol. 5, pp. 371377, 1994.

[35] A.B. Sørensen, "Projective Reed-Muller codes," IEEE Trans. Inform. Theory, vol. IT-37, pp. 1567-1576, Nov. 1991

[36] A.B. Sørensen, "Weighted projective Reed-Muller codes and algebraic geometric codes," IEEE Trans. Inform. Theory, vol. IT-38, pp.1821-1826, 1992. 
[37] H. Stichtenoth, Algebraic function fields and codes, Universitext, Springer-Verlag, Berlin 1993.

[38] M.A. Tsfasman and S.G. Vlădut, Algebraic geometric codes, Mathematics and its Applications, vol. 58, Kluwer Acad, Publ., Dordrecht 1991.

[39] M.A. Tsfasman and S.G. Vlăduţ, "Survey on generalized Hamming weights," IEEE Trans. Inform. Theory, vol. IT-41, pp. 1564-1588, Nov. 1995.

[40] K. Yang, P.V. Kumar and H. Stichtenoth, "On the weight hierarchy of geometric Goppa codes," IEEE Trans. Inform. Theory, vol. IT-40, pp. 913-920, May 1994.

[41] V.K. Wei, "Generalized Hamming weights for linear codes," IEEE Trans. Inform. Theory, vol. IT-37, pp. 1412-1418, Sept. 1991.

[42] V.K. Wei and K. Yang, "On the generalized Hamming weights of product codes," IEEE Trans. Inform. Theory, vol. IT-39, pp. 1709-1713. Sept. 1993.

[43] E.J. Weldon, "New generalizations of the Reed-Muller codes. Part II: nonprimitive codes," IEEE Trans. Inform. Theory, vol. IT-14, pp. 199205, 1968.

[44] X.-W. Wu, "Generalized Hamming weights of some classes of projective codes," Ph.D. Thesis, Institute of System Sciences, Beijing, May 1995. 